\title{
Assimilating compact phase space retrievals (CPSRs): comparison with independent observations (MOZAIC in situ and IASI retrievals) and extension to assimilation of truncated retrieval profiles
}

\author{
Arthur P. Mizzi ${ }^{1, a}$, David P. Edwards ${ }^{1}$, and Jeffrey L. Anderson ${ }^{2}$ \\ ${ }^{1}$ National Center for Atmospheric Research, Atmospheric Chemistry Observations and Modeling Laboratory, \\ Boulder, CO 80305, USA \\ ${ }^{2}$ National Center for Atmospheric Research, Computational and Information Systems Laboratory, Boulder, CO 80305, USA \\ ${ }^{a}$ currently at: Colorado Department of Public Health \& Environment, Air Pollution Control Division, \\ Denver, CO 80246, USA
}

Correspondence: Arthur P. Mizzi (mizzi@ucar.edu)

Received: 25 January 2018 - Discussion started: 2 March 2018

Revised: 10 August 2018 - Accepted: 15 August 2018 - Published: 17 September 2018

\begin{abstract}
Assimilation of atmospheric composition retrievals presents computational challenges due to their high data volume and often sparse information density. Assimilation of compact phase space retrievals (CPSRs) meets those challenges and offers a promising alternative to assimilation of raw retrievals at reduced computational cost (Mizzi et al., 2016). This paper compares analysis and forecast results from assimilation of Terra/Measurement of Pollution in the Troposphere (MOPITT) carbon monoxide (CO) CPSRs with independent observations. We use MetOp-A/Infrared Atmospheric Sounding Interferometer (IASI) CO retrievals and Measurement of OZone, water vapor, carbon monoxide, and nitrogen oxides by in-service AIrbus airCraft (MOZAIC) in situ CO profiles for our independent observation comparisons. Generally, the results confirm that assimilation of MOPITT CPSRs improves the Weather Research and Forecasting model with chemistry coupled to the ensemble Kalman filter data assimilation from the Data Assimilation Research Testbed (WRF-Chem/DART) analysis fit and forecast skill at a reduced computational cost compared to assimilation of raw retrievals. Comparison with the independent observations shows that assimilation of MOPITT CO generally improved the analysis fit and forecast skill in the lower troposphere but degraded it in the upper troposphere. We attribute that degradation to assimilation of MOPITT CO retrievals with a possible bias of $\sim 14 \%$ above $300 \mathrm{hPa}$. To discard
\end{abstract}

the biased retrievals, in this paper, we also extend CPSRs to assimilation of truncated retrieval profiles (as opposed to assimilation of full retrieval profiles). Those results show that not assimilating the biased retrievals (i) resolves the upper tropospheric analysis fit degradation issue and (ii) reduces the impact of assimilating the remaining unbiased retrievals because the total information content and vertical sensitivities are changed.

\section{Introduction}

The adverse impacts of poor air quality on human health and welfare are well documented, e.g., Harvey (2016) and Robichaud (2017). Air quality analyses and forecasts, more generally chemical weather products, are used to help understand and preempt poor air quality events. The accuracy of such chemical weather products depends in part on the application of chemical data assimilation to combine air quality observations with independent estimates of the air quality state to produce an "optimal" chemical weather analysis (Robichaud, 2017). Air chemistry observations generally fall into two categories: in situ and remote. In situ observations come from direct observational platforms like samplers, and remote observations come from indirect observa- 
tional platforms like satellites. Due to the spatial and temporal sparsity of in situ observations, air quality managers and researchers are increasingly relying on satellite observations. Such observations generally come in the form of "retrievals," and their use involves challenges that include (i) low information density (the amount of information per retrieval is small), (ii) large volumes of data, (iii) incorporation of unobserved information from the retrieval prior, and (iv) correlated observation errors (Mizzi et al., 2016). In the chemical weather forecasting/data assimilation literature, there have been several papers that have studied those challenges; see Joiner and Da Silva (2006), Migliorini et al. (2008), and Mizzi et al. (2016). Generally, other researchers have dealt with challenges (i) and (ii) by assimilating all the available retrievals (e.g., Jiang et al., 2015). They have dealt with challenge (iii) by assimilating the contribution from the retrieval priors (e.g., Jiang et al., 2015), and they have dealt with challenge (iv) by ignoring the error correlations (e.g., Barre et al., 2015). As discussed in Mizzi et al. (2016), the problem with their approach for addressing challenges (i) and (ii) is that it is computationally expensive and inefficient to assimilate all the retrievals. Some researchers have tried to address this by discarding (not assimilating) some of the retrievals in the vertical profile (Arellano Jr. et al., 2007). A similar strategy is used by some researchers to address biased retrievals; i.e., they do not assimilate the biased retrievals (Barre et al., 2015). Some of the results in this paper suggest there are unexpected adverse impacts from discarding selected elements and assimilating the remaining elements of a retrieval profile. Mizzi et al. (2016) introduced the assimilation of "compact phase space retrievals" (CPSRs) to address challenges (i) and (ii) without discarding elements of the retrieval profile. In this paper, we extend the CPSR algorithm to truncated retrieval profiles (retrieval profiles where some of the elements of the profile are not assimilated). However, as discussed herein, the assimilation of truncated retrieval profiles gives unexpected results due to role of the averaging kernel in the retrieval forward operator.

Joiner and Da Silva (2006) was the first paper to address challenge (iii) - not assimilating the retrieval prior contribution. They proposed three approaches. In the first, they characterized the retrieval equation:

$\boldsymbol{y}_{\mathrm{r}}=\mathbf{A} \boldsymbol{y}_{\mathrm{t}}+(\mathbf{I}-\mathbf{A}) \boldsymbol{y}_{\mathrm{a}}+\boldsymbol{\varepsilon}$,

where $\boldsymbol{y}_{\mathrm{r}}$ is the retrieval profile (column vector, dimension $n$ - the number of observations in a full retrieval profile), $\mathbf{I}$ is the identity matrix (square matrix, dimension $n \times n), \mathbf{A}$ is the averaging kernel (square matrix, dimension $n \times n$, and rank $k$, where $k<n), \boldsymbol{y}_{\mathrm{a}}$ is the retrieval prior profile (column vector, dimension $n), \boldsymbol{\varepsilon}$ is the measurement error in retrieval space (column vector, dimension $n$ ) with error covariance $\mathbf{E}_{\mathrm{m}}$ (square matrix, dimension $n \times n$ ), and $\boldsymbol{y}_{\mathrm{t}}$ is the unknown true atmospheric profile (column vector, dimension $n)$ as the sum of two linear transformations. The first transformation was a mapping of $\boldsymbol{y}_{\mathrm{t}}$ to retrieval space by $\mathbf{A}$, and the second was a mapping of $\boldsymbol{y}_{\mathrm{a}}$ to retrieval space by $\mathbf{I}-\mathbf{A}$. Then, they projected $\boldsymbol{y}_{\mathrm{r}}$ onto the trailing left singular vectors from a singular value decomposition (SVD) of $\mathbf{I}-\mathbf{A}$. In their second approach, they projected $\boldsymbol{y}_{\mathrm{r}}$ onto the trailing left singular vectors from an SVD of the retrieval prior error as mapped by $\mathbf{I}-\mathbf{A}$. Finally, their third approach proposed a revised retrieval process that eliminated the need for $\boldsymbol{y}_{\mathrm{a}}$. Those approaches were generally successful and introduced the idea of assimilating phase space retrievals. The second paper to address challenge (iii) was Migliorini et al. (2008). They formed the "quasi-optimal retrieval" (QOR) equation by subtracting the $(\mathbf{I}-\mathbf{A})$ term in Eq. (1) from $\boldsymbol{y}_{\mathrm{r}}$ (to remove the prior contribution). Then, to address challenges (i), (ii), and (iv), they projected the result onto the leading left singular vectors from an SVD of $\mathbf{E}_{\mathrm{m}}$ and discarded those modes whose ensemble variance was much smaller than the transformed observation error variance. Their approach was generally successful but did not address why the modes of the observation error covariance should be related to the modes of the QOR. Finally, Mizzi et al. (2018) used QORs to address challenge (iii) and two phase space transforms to address challenges (i), (ii), and (iv). The first was a compression transform based on the leading left singular vectors of A. This step enabled compression because $\mathbf{A}$ is highly rank deficient. Since those singular vectors span the range of $\mathbf{A}$ and the QORs are in that range, their respective modes were mathematically related. The second was a diagonalization transform to account for the observation error covariance during the assimilation. Their approach was generally successful. The Mizzi et al. (2016) and Migliorini et al. (2008) algorithms are different. The Migliorini et al. (2008) approach was motivated by rank deficiency of the observation error covariance and whether the phase space ensemble error variance was small relative to the transformed observation error variance. The Mizzi et al. (2016) approach was motivated by rank deficiency of the averaging kernel and accounting for the observation error covariance. The spaces spanned by the respective transform vectors are different. The Migliorini et al. (2008) vectors spanned observation error covariance space, and the Mizzi et al. (2016) vectors spanned QOR space. The Migliorini et al. (2008) compression was based on the relative magnitude of the transformed ensemble error and observation error variance, and the Mizzi et al. (2016) compression was based on the removal of redundant information for the QOR.

One aspect of assimilating retrievals not addressed by Migliorini et al. (2008) or Mizzi et al. (2016) is how to apply their algorithms when the retrieval profile is truncated. Such an extension is necessary if one wants to assimilate only a portion of the retrieval profile. Both methods can be extended, so one goal of this paper is to document that extension for CPSRs and evaluate the results.

Mizzi et al. (2016) demonstrated the utility of assimilating CPSRs by verifying the analysis and forecast results against the assimilated observations. In this paper, we compare our 
results against both the assimilated and independent observations. As in Mizzi et al. (2016), we assimilate conventional meteorological observations and Terra/Measurement of Pollution in the Troposphere (MOPITT) CO retrievals, but here we also compare our analysis and forecast results with MetOp-A/Infrared Atmospheric Sounding Interferometer (IASI) CO retrievals and Measurement of OZone, water vapor, carbon monoxide, and nitrogen oxides by in-service AIrbus airCraft (MOZAIC) in situ CO profiles. Those comparisons are necessary because they provide an independent assessment of the improved analysis fit and forecast skill. The remainder of this paper is organized as follows: Sect. 2 describes the forecast/data assimilation system together with the assimilated meteorological and chemistry observations. Section 3 describes the independent IASI and MOZAIC observations used in the verification analyses. Section 4 presents descriptions of our experiments, retrieval preprocessing methods, and extension of CPSRs to truncated retrieval profiles. Section 5 compares the results of assimilating MOPITT CO retrievals (full and truncated profiles) with the IASI and MOZAIC CO observations. Finally, Sect. 6 presents a summary of our results and conclusions.

\section{WRF-Chem/DART regional forecasting ensemble data assimilation system: setup and assimilated observations}

For the experiments reported here, we use the WRFChem/DART regional chemical transport/ensemble Kalman filter data assimilation system introduced by Mizzi et al. (2016). WRF-Chem/DART is made up of the Weather Research and Forecasting (WRF) model with chemistry (WRF-Chem) (https://www2.acom.ucar.edu/wrf-chem, last access: 6 September 2018) coupled to the ensemble Kalman filter data assimilation from the Data Assimilation Research Testbed (DART) (http://www.image.ucar.edu/ DAReS/DART/, last access: 6 September 2018; Anderson et al., 2009). WRF-Chem is a regional model that predicts conventional weather together with the transport, mixing, and chemical transformation of atmospheric trace gases and aerosols. DART is an ensemble data assimilation system that uses the ensemble adjustment Kalman filter of Anderson $(2001,2003)$ together with adaptive inflation and localization.

We conduct continuous cycling experiments with $6 \mathrm{~h}$ cycling (00:00, 06:00, 12:00, and 18:00 UTC) for the period 1 June 2008, 00:00 UTC, to 9 June 2008, 18:00 UTC. To facilitate a large number of experiments, we use a reduced ensemble size of 20 members, a horizontal resolution of $100 \mathrm{~km}$ $(101 \times 41$ grid points), and an abbreviated 9-day study period (compared to the 30-day period used in Mizzi et al., 2016). The reduced study period is not thought to negatively impact our results because the WRF-Chem/DART spin-up occurs within the first 48 to $72 \mathrm{~h}$. The WRF-Chem domain extends from $\sim 176$ to $\sim 50^{\circ} \mathrm{W}$ and $\sim 7$ to $\sim 54^{\circ} \mathrm{N}$. We use 34 vertical levels with a model top at $10 \mathrm{hPa}$ and $\sim 15$ levels below $500 \mathrm{hPa}$. We use DART adaptive prior covariance inflation with the recommended settings and DART Gaspari-Cohn localization with a localization radius half-width of $\sim 300 \mathrm{~km}$ in the horizontal (Anderson, 2008). Vertical localization is not used. These are the same settings as used by Mizzi et al. (2016).

The WRF-Chem initial and boundary conditions are derived from the National Oceanic and Atmospheric Administration/National Center for Environmental Prediction (NOAA/NCEP) Global Forecast Model (GFS) $0.5^{\circ} 6 \mathrm{~h}$ forecasts. The WRF Preprocessing System (WPS) interpolates the GFS forecasts to our domain and generates the deterministic boundary conditions. We use the WRF Data Assimilation System (WRFDA) (https:www2.mmm.ucar.edu/wrf/ users/docs/user_guide_V3.9/ARWUsersGuideV3.9.pdf, last access: 6 September 2018; Barker et al., 2012) to generate the initial meteorological ensemble. The chemistry initial and boundary conditions are derived from the Model for Ozone and Related Chemical Tracers: MOZART-4 (MOZART) forecasts, and WRF-Chem utilities are used to interpolate those forecasts to our domain and generate the deterministic chemistry boundary conditions. The emissions and initial chemistry ensembles are generated as described in Mizzi et al. (2016). The ensemble distributions are Gaussian with a specified mean and standard deviation. The tails of those distributions are truncated to include $95 \%$ of the distribution and exclude outliers. That strategy ensures that the emissions and initial chemistry variable concentrations are positive definite. We do not include horizontal correlations for the emission perturbations because they are not relevant to the focus of this paper.

At each cycle time, depending on the experiment, we assimilate conventional meteorological and chemistry observations with DART and advance the analysis ensemble to the next cycle time with WRF-Chem. The resulting $6 \mathrm{~h}$ forecast ensemble is then used as the first guess in the next assimilation step. Our conventional meteorological observations are NCEP automated data processing (ADP) upper air and surface observations (PREPBUFR observations), and our chemistry observations are MOPITT CO mixing ratio retrieval profiles. MOPITT is an instrument on the National Aeronautics and Space Administration's (NASA's) Earth Observing System Terra satellite. Its spatial resolution is $22 \mathrm{~km}$ at nadir over a swath width of $640 \mathrm{~km}$. Its thermal infrared (TIR) measurements are sensitive to $\mathrm{CO}$ in the middle and upper troposphere, while its near-infrared (NIR) measurements are sensitive to total column CO. MOPITT provides global coverage every 3 to 4 days. MOPITT CO is reported on 10 vertical levels starting at a variable surface pressure level and then ranging from 900 to $100 \mathrm{hPa}$ every $100 \mathrm{hPa}$. We assimilate the MOPITT V5 thermal-infrared/near-infrared (TIR/NIR) retrieval products described by Deeter et al. (2013). Validation results suggest that from $400 \mathrm{hPa}$ to the surface the 
MOPITT CO retrievals are accurate to within $5 \%$. Above $400 \mathrm{hPa}$, they may have a positive bias of $\sim 14 \%$ (Deeter et al., 2013, and Martinez-Alonso et al., 2014), that has been addressed in subsequent MOPITT products (Deeter et al., 2014).

The horizontal resolution of the MOPITT data is much greater than that at which we run WRF-Chem. That difference translates to representativeness errors due to the smaller spatial scales that are resolved by the satellite but not by the model. To address those errors, we construct superobservations as follows: (i) sort the retrievals, retrieval priors, averaging kernels, and retrieval error covariances into bins that are $\sim 90 \mathrm{~km}$ square, (ii) calculate the bin average for each of those variables, and (iii) assimilate the bin-average retrievals. We use an arithmetic average (as opposed to an error covariance weighted average) when calculating the superobservations. We do not apply corrections to the retrieval error covariance super-observations because we are interested in the assimilation impact of the reported errors and can apply tuning to those errors and balance the root mean square error (RMSE)/total spread fit as needed. Other studies, e.g., Eskes et al. (2003), Miyazaki et al. (2012a, b, 2015), and Barre et al. (2016), have used similar super-observation strategies. We do not expect that tuning the observation errors would significantly impact our results because our diagnostic analyses showed that the RMSE and total spread were properly balanced.

\section{Independent observations for verification: MOZAIC in situ and IASI CO retrieval profiles}

In the first part of this paper, we compare the analysis and forecast results from assimilating MOPITT $\mathrm{CO}$ with independent observations (IASI CO retrievals and MOZAIC in situ CO profiles). IASI is an instrument on the EUMETSAT (European Organization for the Exploitation of Meteorological Satellites) polar-orbiting MetOp-A satellite (Clerbaux et al., 2009). It measures temperature, water vapor, fractional cloud cover, cloud top temperature, ozone, carbon monoxide, and methane. IASI has been operating from 2006 to the present. Its mission is to provide observational support for numerical weather prediction. IASI measures $\mathrm{CO}$ radiances under cloud-free conditions with a horizontal resolution of $25 \mathrm{~km}$ over a swath width of $\sim 2200 \mathrm{~km}$. IASI measurements are sensitive to $\mathrm{CO}$ in the middle to lower troposphere. IASI provides global coverage every 2 days. IASI $\mathrm{CO}$ is reported on 19 altitude levels ranging from the surface to $18 \mathrm{~km}$ every $1 \mathrm{~km}$. Validation results suggest that the $\mathrm{CO}$ retrievals are accurate to within $13 \%$. For more information, see https://www.eumetsat.int/ (last access: 6 September 2018).

MOZAIC was a European Research Infrastructure (ERI) project that collected long-term, global-scale measurements of atmospheric composition on international commercial air- line flights from August 1994 to November 2014 (Marenco et al., 1998). MOZAIC collected in situ measurement of ozone, water vapor, carbon monoxide, and total nitrogen oxides. The available data products are geolocated (come with longitude, latitude, and pressure coordinates) and include simultaneous meteorological observations. During MOZAIC, data acquisition was automatically performed on the ascent, descent, and cruise phases of round-trip international flights between Europe and the US, Africa, the Middle East, and Asia. For more information, see http://www.iagos.fr/ (last access: 6 September 2018).

\section{Experimental design}

We conduct WRF-Chem/DART forecast/assimilation cycling experiments that are similar to those of Mizzi et al. (2016). The primary differences are the (i) use of superobservations, (ii) extension of CPSRs to truncated retrieval profiles, and (iii) use of localization to preclude the assimilated MOPITT CO observations from impacting any state variable other than $\mathrm{CO}$. We performed a control experiment where we assimilated only conventional meteorological observations (the MET experiment), and we performed a series of chemical data assimilation experiments. In those experiments, we studied assimilation results from four types of retrieval preprocessing strategies: (i) volume mixing ratio retrievals (VMRRs, the associated experiment is called the VMRR experiment), (ii) $\log _{10}$ (VMRR) retrievals (L10VMRRs, the L10VMRR experiment), (iii) compact phase space retrievals (CPSRs, the CPSR experiment), and (iv) quasi-optimal retrievals (QORs, the QOR experiment). The CPSR and QOR experiments (as applied to assimilation of full retrieval profiles) were studied by Mizzi et al. (2016). The VMRR experiment and the L10VMRR and CPSR experiments as applied to assimilation of truncated retrieval profiles are new. We include the L10VMR and QOR experiments as applied to retrieval full profiles because, as discussed in the introduction, our comparison of those experiments with independent observations (discussed below in Sect. 5.1) suggests that it may be beneficial to not assimilate MOPITT CO retrievals in the upper troposphere due to their possible bias. That concern motivates application of the L10VRR and CPSR experiments to the assimilation of truncated retrieval profiles. The rest of this section describes those experiments. It should be noted that the different retrieval preprocessing methods (making up the different experiments) are applied after the customary quality assurance/quality control (QA/QC) checks that might discard entire retrieval profiles. Those forecast/assimilation experiments are summarized in Table 1. 
Table 1. Summary of the WRF-Chem/DART forecast/data assimilation experiments.

\begin{tabular}{lcccccc}
\hline Experiment & $\begin{array}{c}\text { Assimilate } \\
\text { meteorology } \\
\text { observations }\end{array}$ & $\begin{array}{c}\text { Assimilate } \\
\text { raw MOPIT } \\
\text { retrievals }\end{array}$ & $\begin{array}{c}\text { Assimilate } \\
\text { MOPITT } \\
\text { CPSRs }\end{array}$ & $\begin{array}{c}\text { Assimilate } \\
\text { MOPITT } \\
\text { QORs }\end{array}$ & $\begin{array}{c}\text { Assimilate } \\
\text { full retrieval } \\
\text { profiles }\end{array}$ & $\begin{array}{c}\text { Assimilate } \\
\text { retrieval truncated } \\
\text { profiles }\end{array}$ \\
\hline MET & Yes & No & No & No & No & No \\
VMRR & Yes & Yes & No & No & Yes & No \\
L10VMRR & Yes & Yes & No & No & Yes & No \\
CPSR & Yes & No & Yes & No & Yes & No \\
QOR & Yes & No & No & Yes & Yes & No \\
L10VMRR-RJ3 & Yes & Yes & No & No & No & Yes \\
CPSR-RJ3 & Yes & No & Yes & No & No & Yes \\
\hline
\end{tabular}

\subsection{The VMRR and L10VMRR experiments}

The MOPITT CO retrieval, averaging kernel, and error covariance products are reported in units of $\log _{10}(\mathrm{VMR})$. The IASI CO products are in VMR. For ease of comparison and interpretation, it is convenient to convert the MOPITT data from L10VMRR to VMRR. While it is possible to convert the retrievals and error covariance, it is not possible to convert the averaging kernels. Consequently, for the VMRR experiment, the DART forward operator for MOPITT CO converts the state space CO profile from VMRRs to L10VMRRs, applies the averaging kernel, and then converts the resulting expected observation (the expected retrieval profile) to VMR. For the L10VMRR experiment, a conversion is not necessary because the state space CO profile is in $\log _{10}(\mathrm{VMR})$. Conceptually, we expect little difference between the VMRR and L10VMRR experiments due to an underlying assumption that L10VMRRs have a Gaussian distribution and the VMRRs have a lognormal distribution (Deeter et al., 2007a). However, non-linearity of the base-10 exponential operator that relates the L10VMRRs to the VMRRs and the extent to which the VMRR distributions are non-Gaussian may introduce differences. So, one goal of the related experiments is to determine whether those differences are significant. Another reason is to include preprocessing methods that enable us to not assimilate selected retrievals so we can compare the assimilation/forecast results with those from applying CPSRs to truncated retrieval profiles.

\subsection{The QOR experiment}

The assimilation of QORs was discussed in Mizzi et al. (2016). We include QOR assimilation/forecast experiments for completeness and to provide a reference against which to compare the other retrieval preprocessing experiments. In addition (although not discussed herein), QOR preprocessing can be applied to truncated retrieval profiles using the extension discussed in the next section on the CPSR experiment.

QORs are retrieval residuals introduced by Migliorini et al. (2008). They are derived by writing the retrieval equation as

$\boldsymbol{y}_{\mathrm{r}}-(\mathbf{I}-\mathbf{A}) \boldsymbol{y}_{\mathrm{a}}-\boldsymbol{\varepsilon}=\mathbf{A} \boldsymbol{y}_{\mathrm{t}}$,

and transforming Eq. (2) with the left singular vectors from the SVD of $\mathbf{E}_{\mathrm{m}}$ divided by the square root of the associated singular value. If the SVD of $\mathbf{E}_{\mathrm{m}}$ is $\mathbf{E}_{\mathrm{m}}=\phi \sigma \varphi^{T}$, then the QOR profile is defined as

$\sigma^{-1 / 2} \phi^{T}\left(\boldsymbol{y}_{\mathrm{r}}-(\mathbf{I}-\mathbf{A}) \boldsymbol{y}_{\mathrm{a}}-\varepsilon\right)=\sigma^{-1 / 2} \phi^{T} \mathbf{A} \boldsymbol{y}_{\mathrm{t}}$,

and the transformed $\mathbf{E}_{\mathrm{m}}$ is the identity matrix. That transform is similar to the CPSR diagonalization transform described in the next section, except Migliorini et al. (2008) applied the QOR transform to the raw averaging kernel and the raw error covariance while Mizzi et al. (2016) applied it to the compressed averaging kernel and the compressed error covariance. In our application of QORs, there is no filtering of the dominant modes. Also, in general, the QOR transform has no zero singular values because $\mathbf{E}_{\mathrm{m}}$ is not singular.

\subsection{The CPSR experiment and the extension of CPSRs to assimilation of truncated retrieval profiles}

The derivation and assimilation of CPSRs was introduced by Mizzi et al. (2016). They derived CPSRs by applying two transforms to Eq. (2): (i) a compression transform based on the SVD of $\mathbf{A}$, and (ii) a diagonalization transform based on the SVD of the compressed $\mathbf{E}_{\mathrm{m}}$. Their application can be characterized as CPSRs applied to full retrieval profiles (because none of the elements in the retrieval profile were discarded) or to square systems (because $\mathbf{A}$ is a square matrix). If we discard one or more elements of $\boldsymbol{y}_{\mathrm{r}}$, then we must also discard the corresponding rows of $\mathbf{A}$ (call the modified forms $\hat{y}_{\mathrm{r}}$ and $\hat{A}$, respectively). The resulting $\hat{A}$ is not a square matrix. Note that we must also discard the corresponding rows and columns of $\mathbf{E}_{\mathrm{m}}$, so it remains square but its dimension is reduced. This application can be characterized as CPSRs applied to truncated retrieval profiles (because some of the elements of the retrieval profile have been discarded) or to rectangular systems (because $\hat{A}$ is a non-square rectangular matrix). The mathematical formalism for CPSRs applied to 
rectangular systems is the same as that for square systems because Mizzi et al. (2016) used a SVD (as opposed to an eigenvalue decomposition) in their derivation. In the remainder of this section, we extend the derivation of CPSRs from Mizzi et al. (2016) to rectangular systems.

We begin by conceptually discarding $q$ elements of $\boldsymbol{y}_{\mathrm{r}}$. Generally, we discard the elements of the full retrieval profile $\boldsymbol{y}_{\mathrm{r}}$ that are known to be systematically bad observations. If we discard multiple elements, they need not be sequential. The resulting truncated retrieval profile is denoted $\hat{y}_{\mathrm{r}}$ and its dimension is $\hat{n}=n-q$. We must also discard (i) the corresponding elements of $\varepsilon$ to get $\hat{\varepsilon}$ with dimension $\hat{n}$, (ii) the corresponding rows of $\mathbf{A}$ to get $\hat{A}$ with dimension $\hat{n} \times n$, and (iii) the corresponding rows and columns of $\mathbf{E}_{\mathrm{m}}$ to get $\hat{E}_{\mathrm{m}}$ with dimension $\hat{n} \times \hat{n}$. Without loss of generality, we can drop the ${ }^{-}$notation for the remainder of this paper and let $\boldsymbol{y}_{\mathrm{r}}, \boldsymbol{\varepsilon}$, $\mathbf{A}$, and $\mathbf{E}_{\mathrm{m}}$ represent their respective terms before and after discarding the retrieval elements that will not be assimilated. The rest of the derivation is the same as in Mizzi et al. (2016).

First, we apply the compression transform based on the leading left singular vectors of $\mathbf{A}$. If $\mathbf{A}=\mathbf{U S V}^{T}$ is the SVD and $A_{0}=U_{0} S_{0} V_{0}^{T}$ is the truncated SVD where the trailing singular vectors (those whose singular values are less than an ad hoc threshold of $1.0 \times 10^{-4}$ ) are replaced with zero vectors and the trailing singular values are set to zero, then the compressed form of Eq. (2) is

$\mathbf{U}_{0}^{T}\left(\boldsymbol{y}_{\mathrm{r}}-(\mathbf{I}-\mathbf{A}) \boldsymbol{y}_{\mathrm{a}}-\boldsymbol{\varepsilon}\right)=\mathbf{S}_{0} \mathbf{V}_{0}^{T} \boldsymbol{y}_{\mathrm{t}}$

and the compressed error covariance is

$\mathbf{U}_{0}^{T} \mathbf{E}_{\mathrm{m}} \mathbf{U}_{0}$.

In that step, there is no filtering of the dominant modes. Next, we apply the diagonalization transform. If the SVD of the compressed error covariance in Eq. (5) is $\mathbf{U}_{0}^{T} \mathbf{E}_{\mathrm{m}} \mathbf{U}_{0}=$ $\Phi \Sigma \boldsymbol{\Psi}^{T}$, then the diagonalized and conditioned form of Eq. (4) is

$\boldsymbol{\Sigma}^{-1 / 2} \boldsymbol{\Phi}^{T} \mathbf{U}_{0}^{T}\left(\boldsymbol{y}_{\mathrm{r}}-(\mathbf{I}-\mathbf{A}) \boldsymbol{y}_{\mathrm{a}}-\boldsymbol{\varepsilon}\right)=\boldsymbol{\Sigma}^{-1 / 2} \boldsymbol{\Phi}^{T} \mathbf{S}_{0} \mathbf{V}_{0}^{T} \boldsymbol{y}_{\mathrm{t}}$,

and that of Eq. (5) is the identity matrix. Equations (4)-(6) and the fully transformed error covariance are the same as in Mizzi et al. (2016) except that unwanted retrieval elements have been discarded.

Finally, we note that the rank of $\mathbf{A}$ and the rank of $\hat{A}$ are generally the same provided the difference between the dimension of $\mathbf{A}$ and the rank of $\mathbf{A}$ is greater than or equal to the number of discarded elements from the retrieval profile, i.e., $n-k \geq q$. That statement is not necessarily true, but given the rank deficiency of $\mathbf{A}$ it is usually true. We also note that the $\boldsymbol{\Sigma}^{-1 / 2} \boldsymbol{\Phi}^{T} \mathbf{S}_{0} \mathbf{V}_{0}^{T}$ on the right side of Eq. (6) is the transformed averaging kernel. It represents the sensitivity of the phase space retrievals (the CPSRs) to the true CO concentrations at each vertical level. Unlike the raw averaging kernel, which included sensitivities to the null space contributions to the retrieval (the linearly dependent contributions from the right side of Eq. 2), the transformed averaging kernel contains only sensitivities for the measurement contributions to the retrieval (the linearly independent contributions from the right side of Eq. 2).

\section{Results}

\subsection{Assimilation of full retrieval profiles}

In this section, we look at assimilation/forecast results from the experiments described in Sect. 4. The reader should note that the CPSR and QOR experiments are the same as the MOP CPSR and MOP QOR experiments from Mizzi et al. (2016) except (i) the study period is shorter (9 days as opposed to 1 month), (ii) we assimilate MOPITT superobservations, and (iii) we use localization to preclude the assimilated MOPITT CO observations from impacting any state variable other than $\mathrm{CO}$.

Figure 1 shows forecast verification statistics (RMSE and bias) for the different experiments when compared against the assimilated MOPITT CO retrievals on the left and the independent IASI CO retrievals on the right. For the MOPITT comparison, the MOPITT CO forward operator has been applied to the WRF-Chem results so the comparison is made in MOPITT CO retrieval space. Similarly, for the IASI comparison, the IASI CO forward operator has been applied so the comparison is made in IASI CO retrieval space. The left panel can be compared with Fig. 8 from Mizzi et al. (2016). Qualitatively, that comparison shows that the two figures are similar. The MET experiment yields the highest RMSE and bias, while the CPSR and QOR experiments yield lower RMSE and bias. Similar results are seen in the IASI CO comparison. It is interesting that for both comparisons (i) the VMRR experiment shows a slight degradation when compared to the MET experiment, and (ii) the VMRR and L10VMRR experiments are similar to the MET experiment. We suspect that result (i) is a consequence of the nonlinearity of the base-10 log function and the non-Gaussianity of the VMRR distributions, and result (ii) is a consequence of the magnitude of the observation errors used in the VMRR and L10VMRR experiments (discarding the observation error cross-covariance produced observation error variances that are large compared to those produced by the CPSR diagonalization transform) and the length of the study period. We believe the CPSR observation errors are smaller due to the compression step of the CPSR transform. They cannot be smaller due to the diagonalization step because that is a variance maximizing rotation. So, if the compression had no filtering effect on the errors, the variance resulting from the diagonalization step would no smaller than that from the compression step. One consequence of relatively large observation errors is that it takes more cycles for the assimilation to show an impact. We have run similar experiments with a 

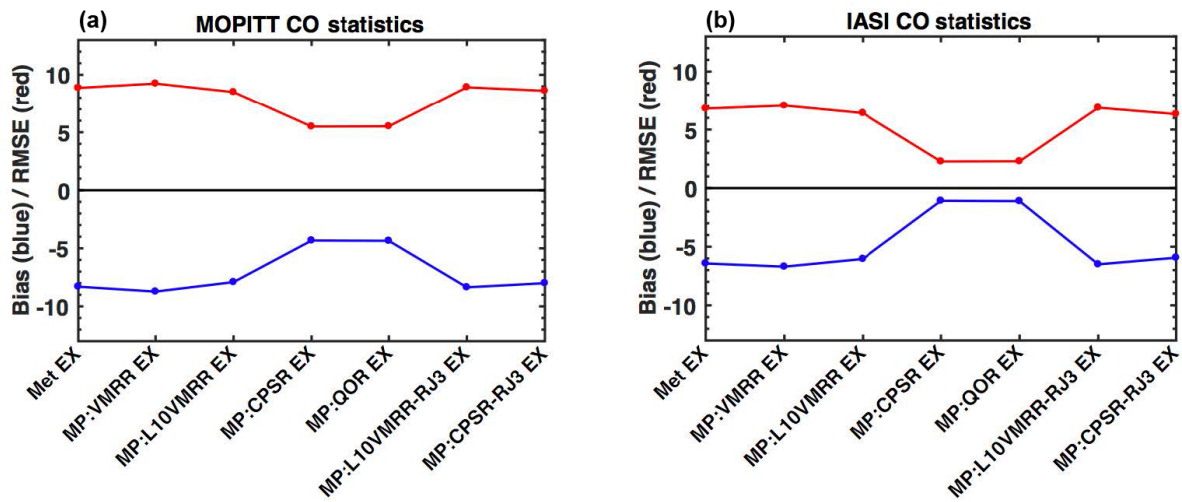

Figure 1. Forecast (assimilation prior) verification statistics for all experiments in MOPITT retrieval space on the left and IASI retrieval space on the right. The red curve is root mean square error (RMSE), and the blue curve is bias (model - observation). The experiments are described in the text and summarized in Table 1.

longer study period and found assimilation impacts. We do not view that as a deficiency in the experimental design. We are interested in the assimilation of CPSRs. If they show an impact during a shorter study period but more conventional methods that do not account for redundant information or error correlations fail to show an impact, then that failure identifies deficiencies in the conventional methods.

Figure 1 generally shows increasing improvement when moving from the MET to L10VMRR to CPSR and QOR experiments. As discussed previously, the VMRR and L10VMRR experiments show little to no improvement over the MET experiment. In Fig. 1, the CPSR and QOR experiments show comparable skill. That result can also be seen in Mizzi et al. (2016) by comparing Figs. 3 and 7. There are two potential explanations. First, we use the retrieval space retrieval error covariance $\left(\mathbf{E}_{\mathrm{r}}\right)$ as the observation error covariance to account for other unquantified error sources, and $\mathbf{E}_{\mathrm{r}}=(\mathbf{I}-\mathbf{A}) \mathbf{E}_{\mathrm{a}}$, where $\mathbf{E}_{\mathrm{a}}$ is the retrieval a priori error covariance. If the singular vectors of $\mathbf{E}_{\mathrm{r}}$ are equivalent to those of $\mathbf{A}$, we would get similar results from the CPSR and QOR experiments. However, $\mathbf{E}_{\mathrm{a}}$ is specified in the retrieval algorithm as a covariance matrix, and generally there is no reason to suspect that the singular vectors of $\mathbf{E}_{\mathrm{r}}$ are equivalent to those of $\mathbf{A}$ (for MOPITT CO, they are not equivalent because their respective singular vectors are not orthogonal). Second, in the QOR experiment, the diagonalization transform rotates the QOR equation so that the observation error cross-covariance contributions for each mode are included in their corresponding observation error variance. However, those modes are linearly dependent in the space defined by the rotated averaging kernel because the rotated averaging kernel is still singular. When those linearly dependent modes are assimilated, there is very little adjustment to the analysis. Consequently, the CPSR and QOR experiments yield similar results because (i) the QOR experiment apportions the error and assimilates the linearly dependent modes (which have little or no impact), while (ii) the CPSR experiment appor- tions the error and does not assimilate the linearly dependent modes. Those results differ from the VMRR and L10VMRR experiments because the observation error variance used in the retrieval space experiments does not account for the error cross-covariance contributions, and the linearly independent portion of that error is different from that in the CPSR and QOR experiments.

In Fig. 2, we compare results from the CPSR and MET experiments with the MOZAIC ascent and descent soundings for Dallas, TX (two soundings composited), Portland, OR (four soundings composited), and Philadelphia, PA (two soundings composited). The MOZAIC soundings from 1 June 2008 (Dallas, TX) were discarded because they were observed during our spin-up period. Otherwise, out MOZAIC comparisons were not impacted by forecast/assimilation system spin-up. No other MOZAIC soundings were available for our study period and domain. The MOZAIC soundings used in Fig. 2 were generally not spatially (within several hundred kilometers) or temporally (within $3 \mathrm{~h}$ ) coincident with the MOPITT observations. We linearly interpolated the WRF-Chem forecasts to the MOZAIC observation times and locations and then composited the results. We did not plot the composited MOZAIC profile below $750 \mathrm{hPa}$ because those data are more representative of the lower troposphere over urban areas than are our model grid and assimilated super-observations. The MOZAIC comparison results are qualitatively similar to those from Fig. 1. The CPSR experiment shows that (i) assimilation of phase space retrievals improves the $6 \mathrm{~h}$ forecast skill in the middle and lower troposphere when compared to the MET experiment for Dallas, TX, and Portland, OR, but degrades the skill in the upper troposphere; (ii) assimilation generally degrades skill throughout the troposphere for Philadelphia, PA; (iii) none of the assimilation impacts are significant based on the ensemble variability; and (iv) assimilation provides little or no change near the surface. The upper tropospheric degradation in results (i) and (ii) is related to the 


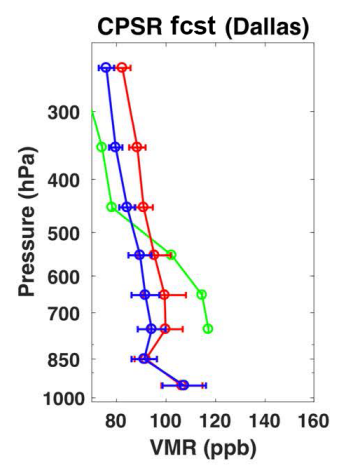

(a)

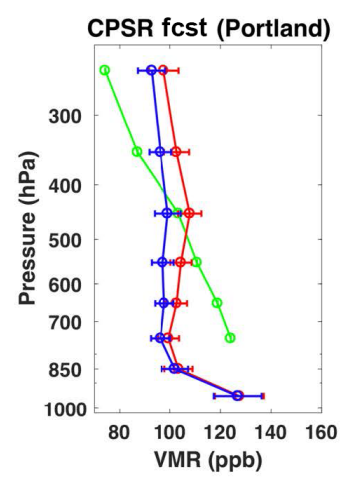

(b)

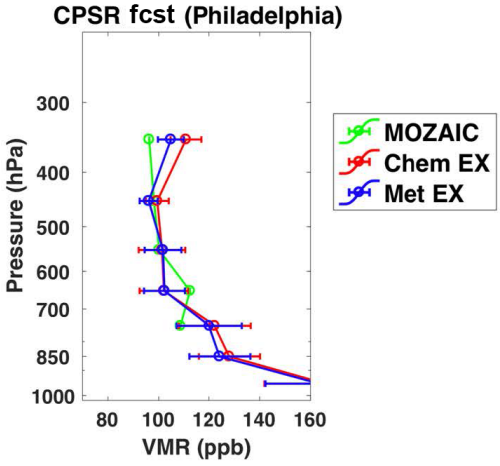

(c)

Figure 2. Comparisons of the CPSR experiment against the IAGOS/MOZAIC in situ CO profiles in ppb composited for 1 June 2008 for Dallas, TX, in panel (a), 3 and 9 June 2008 for Portland, OR, in panel (b), and 7 June 2008 for Philadelphia, PA, in panel (c). Chem EX refers to the CPSR experiment. The error bars are based on the ensemble variability.

positive bias in upper tropospheric MOPITT retrievals discussed earlier. Result (iii) is likely a result of the small sample size, but given the magnitude of the skill differences in the middle and upper troposphere and the "near significance" suggested by some of the error bars, we think there is value in presenting these results. Result (iv) is somewhat unexpected because MOPITT retrievals are documented to have sensitivity to $\mathrm{CO}$ in the upper and lower troposphere (Deeter et al., 2007b). Also, other chemical data assimilation researchers, e.g., Jiang et al. (2013) and Barre et al. (2015), have reported near-surface improvements due to assimilation of MOPITT $\mathrm{CO}$ multispectral retrievals. We suspect result (iv) occurs because MOPITT's upper tropospheric sensitivities dominate its lower tropospheric sensitivities in the transformed system.

To test that hypothesis, we plot a histogram of the MOPITT degrees of freedom for signal (DOFS) for all terrestrial profiles in our domain during the study period in Fig. 3. The MOPITT DOFS is a measure of the amount of independent observed information in a retrieval profile. If a profile contains independent information from the upper and lower troposphere, its DOFS must be $\sim 2.0$. The central histogram of Fig. 3 shows that the mean, median, and mode of DOFSs during this period are $\sim 1.5$ and that DOFSs greater than $\sim 2.0$ are relatively rare $(<5 \%)$. To gain a better understanding of the vertical structure of the MOPITT retrieval information content, we present a composite analysis for averaging kernel profiles in the neighborhood of different DOFS values in the lower row of Fig. 3 where panel (a) is the composite averaging kernels for all DOFS, panel (b) is for $(0.9<$ DOFS $<1.1, \sim 10 \%$ of the histogram probability mass), panel (c) is for $(1.4<$ DOFS $<1.6, \sim 26 \%$ ), and panel (d) is for $(1.9<$ DOFS $<2.1, \sim 4 \%)$. Those panels show that the dominant sensitivity appears to be to the upper troposphere and that as the DOFS approaches 2.0 the sensitivity to the lower troposphere increases. That sensitivity distribution could explain the improvement drop off in the lower troposphere for the MOZAIC comparisons because
Table 2. Average information content for each mode of the averaging kernel for the entire study period. CompAK 1 denotes the average information in mode 1 , CompAK 2 is for mode 2, and so forth. Trace denotes the total information content. "Full histogram" means all retrievals were considered. DOFS denotes the degree of freedom for the signal, and the different DOFS rows identify the average information content for the different DOFS ranges and averaging kernel modes.

\begin{tabular}{lrrrr}
\hline & CompAK 1 & CompAK 2 & CompAK 3 & Trace \\
\hline Full histogram & 0.9638 & 0.4785 & 0.0099 & 1.452 \\
$0.9 \leq$ DOFS $\leq 1.1$ & 0.8997 & 0.1174 & 0.0006 & 1.018 \\
$1.4 \leq$ DOFS $\leq 1.6$ & 0.9771 & 0.5188 & 0.0059 & 1.502 \\
$1.9 \leq$ DOF $\leq 2.1$ & 1.016 & 0.8899 & 0.0518 & 1.957 \\
\hline
\end{tabular}

retrievals with sensitivity to the lower troposphere are relatively rare. However, linear dependencies in the composite averaging kernels of Fig. 3 can mask the significance of the sensitivities to the lower troposphere in the more common DOFS categories.

To unmask those sensitivities, Fig. 4 presents a composite analysis of the different DOFS sensitivities based on the CPSR compression and diagonalization transforms, and Table 2 presents the total and modal information content associated with Fig. 4. The upper row of Fig. 4 shows composite vertical profiles of the leading left singular vectors of the averaging kernel. Those singular vectors (i) span the range of the averaging kernel (the QOR space), (ii) are ranked such that the first singular vector explains the greatest amount of vertical variability in the QOR profile, the second singular vector explains the next greatest amount of variability, and so forth, and (iii) have arbitrary sign, so we chose the sign that has the greatest physical meaning; i.e., we apply a -1.0 scaling to the first and second rows of Fig. 4. Table 2 shows that for $0.9 \leq$ DOFS $\leq 1.0$ most of the information is in the first mode, for $1.4 \leq$ DOFS $\leq 1.5$ two-thirds of the information is in the first mode and one-third is in the second mode, 


\section{MOPITT DOFS HISTOGRAMS}

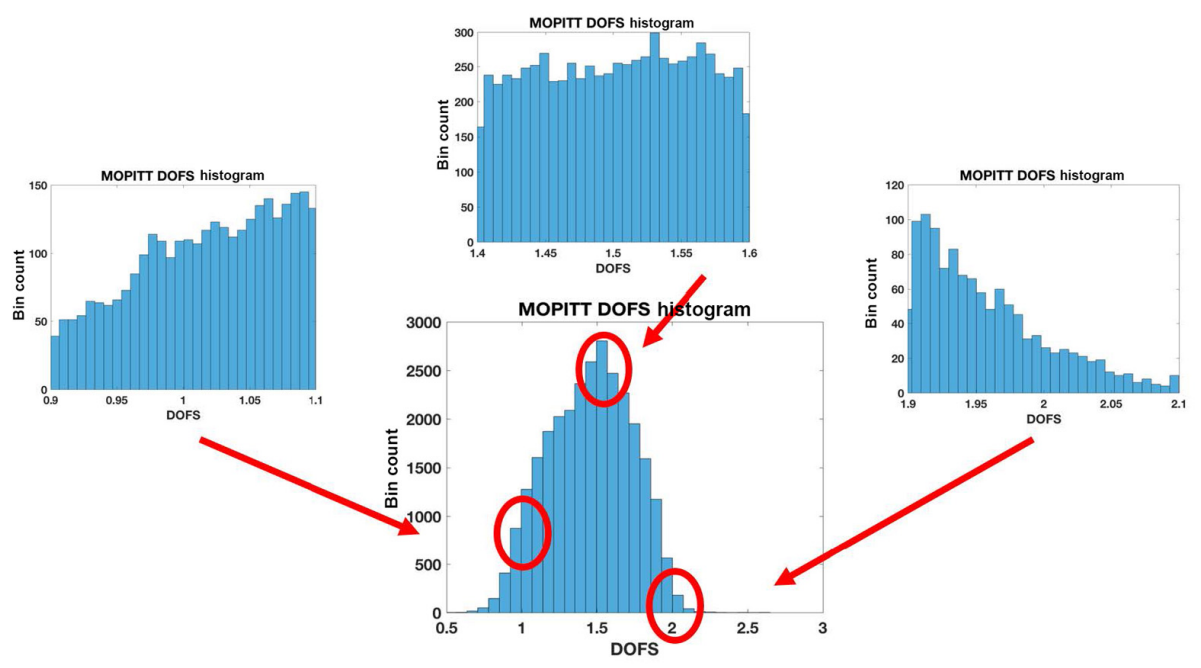

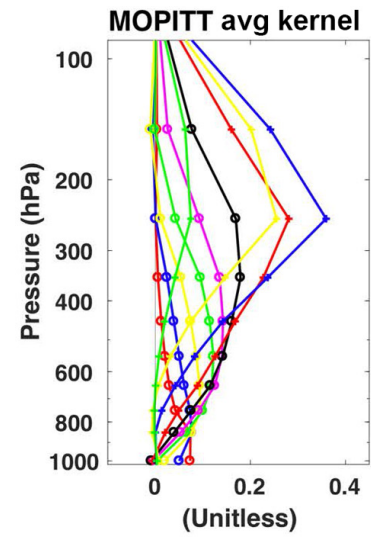

(a)

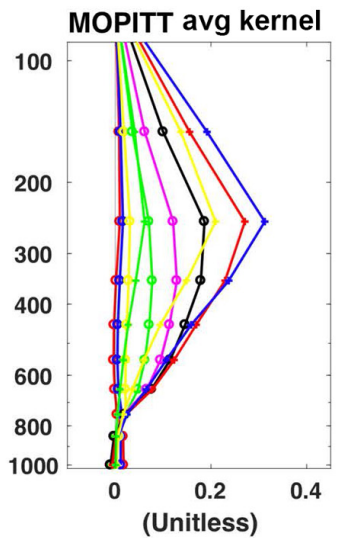

(b)

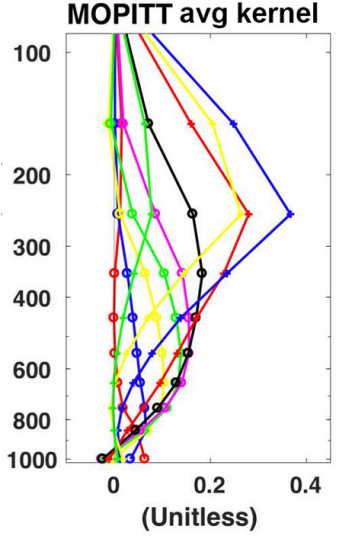

(c)

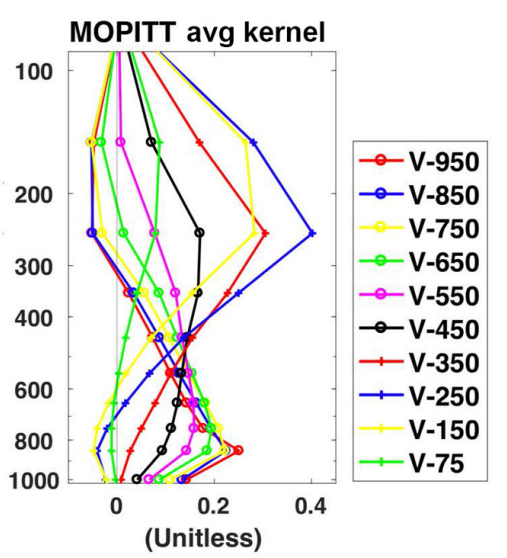

(d)

Figure 3. Histogram of MOPITT CO degrees of freedom of signal (DOFS) with blow-up histograms for selected DOFS ranges in the upper panels. The lower panels show composite MOPITT CO averaging kernel profiles for (a) all DOFS, (b) $(0.9 \leq \mathrm{DOFS} \leq 1.1)$, (c) $(1.4 \leq$ DOFS $\leq 1.6)$, and (d) $(1.9 \leq$ DOFS $\leq 2.1)$. The averaging kernel identifiers are V-xxx where $\mathrm{xxx}$ is the approximate pressure level midpoint in $\mathrm{hPa}$ for the associated averaging kernel profile.

and for $1.9 \leq$ DOFS $\leq 2.1$ one-half of the information is in the first mode and one-half is in the second mode. In Fig. 4, we retained three singular vectors for completeness, but it should be remembered the third vector (and sometimes the second vector) may map information to the null space of the transformed averaging kernel. The second row of Fig. 4 shows composite vertical profiles for the compressed averaging kernels. These profiles show the vertical sensitivity of compressed QORs to the true atmospheric state. The bottom row shows the composite vertical profiles for the compressed and rotated averaging kernels (the profiles after the full CPSR transformation). These profiles show the vertical sensitivity of CPSRs to the true atmospheric state.

Figure 4 shows some interesting results. The upper row of Fig. 4 shows that for DOFS $\approx 1.0$ (column b) the first leading singular vector has positive sensitivity near the surface and negative sensitivity in the middle to upper troposphere (remember that the second and third leading vectors may map to the null space for DOFS $\approx 1.0$ ). As the DOFS increases to 1.5 , the first and second leading vectors have positive sensitivity near the surface and weakly negative sensitivity in the middle to upper troposphere, and for DOFS of 2.0, the first leading vectors have positive sensitivity throughout the troposphere, while the second leading vectors have positive sensitivity near the surface and negative sensitivity in the middle to upper troposphere.

After applying the CPSR diagonalization transform, the DOFS-dependent sensitivity patterns in the second row of Fig. 4 change, and the final patterns (those of the compressed averaging kernels) are shown in the bottom row. These pro- 


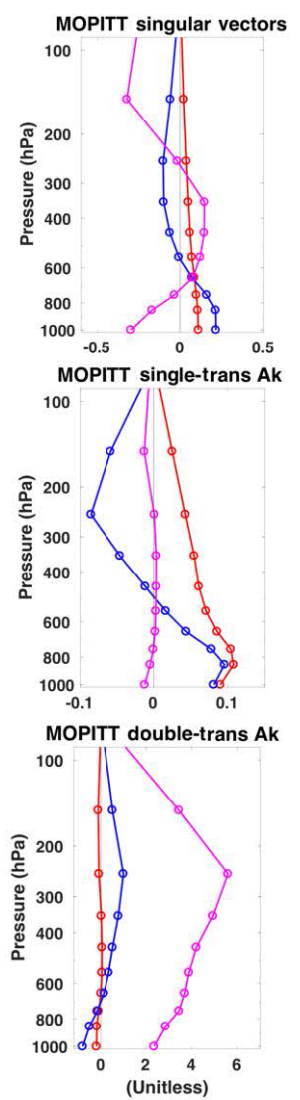

(a)

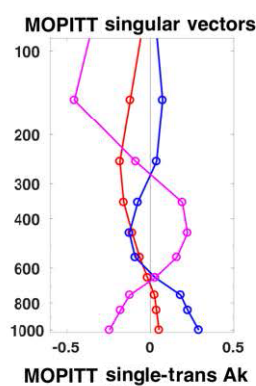

100
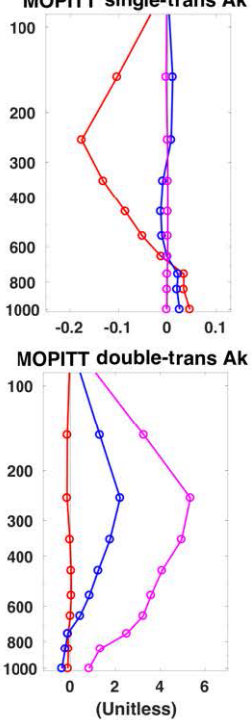

(b)
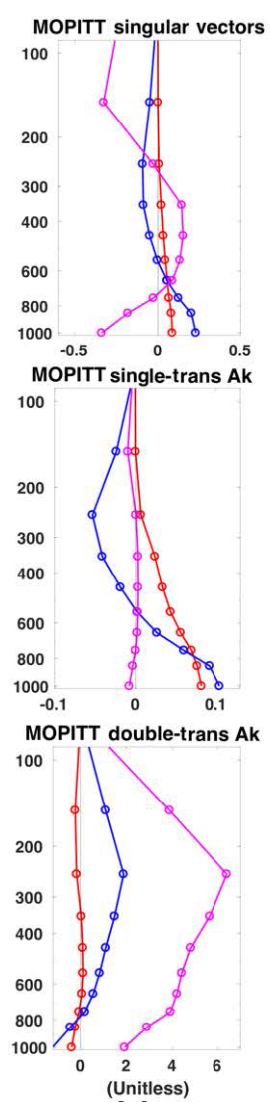

(c)

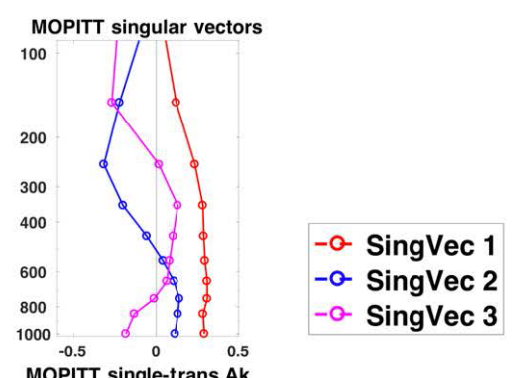

100
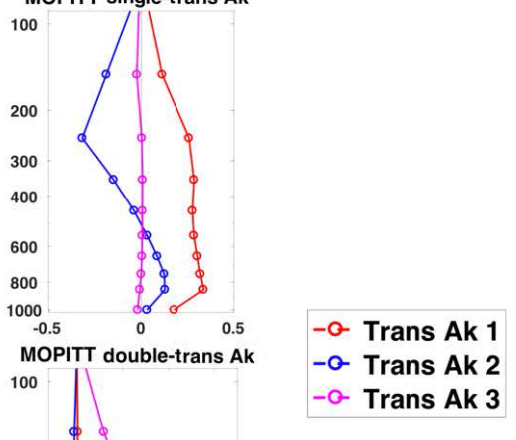

- - Trans Ak 3

Figure 4. Composite vertical profiles for the (i) leading left singular vectors of the MOPITT CO averaging kernels in the upper row, (ii) compressed averaging kernels in the middle row, and (iii) rotated and compressed averaging kernels in the lower row. The DOFS ranges are the same as defined for Fig. 2. For the profile labels, "SingVec x" refers to ranked singular vectors, where $\mathrm{x}=1$ is the first leading singular vector, $\mathrm{x}=2$ is the second leading singular vector, and so forth. "Trans Ak x" refers to the compressed or rotated and compressed averaging kernel profile associated with the QOR and CPSR mode $x$, respectively.

files show that for all DOFS (column a) the first leading mode has its greatest sensitivity near the surface and the sensitivity decreases to a near-zero positive minimum in the upper troposphere. Similarly, the second leading mode has it greatest positive sensitivity near the surface but has strong negative sensitivity in the upper troposphere. The right three columns of the second row in Fig. 4 show the dependency of the vertical sensitivity on the DOFS for the compressed QORs. As seen with the singular vectors, as the DOFS increases the sensitivity changes from weak positive sensitivity near the surface and strong negative sensitivity in the upper troposphere to strong positive sensitivity throughout the troposphere for the first leading mode and positive sensitivity near the surface and strong negative sensitivity in the upper troposphere for the second leading mode. Those results suggest that the MOPITT retrievals (and therefore the results in Fig. 2) should be sensitive to $\mathrm{CO}$ in the lower troposphere/near the surface. However, an interesting thing happens when we account for the reported retrieval error covariance. The lower row of Fig. 4 shows the compressed and rotated averaging kernel profiles, which account for that error covariance. Here, the negative scaling cancels each other because the SVD has been applied twice. These results show first that the significance of the leading modes becomes reversed due to diagonalization transform and scaling by the inverse square root of the compressed and rotated error variance. This does not mean that the third leading mode from the first two rows of Fig. 4 becomes a dominant mode because it may still be mapping to the null space; i.e., the leading CPSR modes (those with the smaller observational error variance) may be mapping to the null space, and the trailing CPSR modes are mapping to the domain of the transformed averaging kernel. That suggests that there may be benefit to not assimilating some of the leading CPSR modes which would be similar to not assimilating the phase space modes with small observational error as was done by Migliorini et al. (2008). The bottom row of Fig. 4 shows that after removing the linear dependencies and accounting for the observation errors, the compressed and rotated averaging kernel has its greatest sensitivity in the upper troposphere for DOFS $<2.0$ and 
weakest sensitivity near the surface for DOFS $\approx 2.0$. That explains why our comparison of the CPSR experiment with the MOZAIC observations in Fig. 2 did not show assimilation impacts near the surface. Other researchers who have assimilated MOPITT CO could not have found this result because they did not adjust for the averaging kernel linear dependencies or for the observation error covariance (see, e.g., Jiang et al., 2015, and Barre et al., 2015).

Figures 5 and 6 show contour maps comparing the MET and CPSR experiments for 9 June 2008, 18:00 UTC (Fig. 5), as well as the assimilated MOPITT and independent IASI $\mathrm{CO}$ retrievals (Fig. 6). Examination of the forecast maps in the upper panel and the forecast difference map (CPSR experiment minus MET experiment) in the lower left panel of Fig. 5 (defined as CPSR EX CO Del-Fcst) shows that assimilation of MOPITT CO retrievals increased the CO concentrations over some areas (southern California, southern Baja, and northern Atlantic east of New England) and decreased the concentrations over broader areas (central to northeastern United States, southeastern United States, and southern Gulf of Mexico). Comparison of the MOPITT CO retrievals in the upper panels of Fig. 6 (the assimilated retrievals) with Fig. 5 shows that the analysis and forecast impacts are generally consistent with the observations. Over southern Baja, the MOPITT observations in Fig. 6 report $\mathrm{CO}$ on the order of $50 \mathrm{ppb}$, while the forecast in Fig. 5 (the assimilation prior) reports $\mathrm{CO}$ on the order of $100 \mathrm{ppb}$. The assimilation increment shows a CO reduction (consistent with the MOPITT observations) on the order of $50 \mathrm{ppb}$. Similarly, the increased CO in the central United States, over Kansas and Nebraska, and in the southeastern United States near Georgia, South Carolina, and Virginia (highlighted by the analysis increment map in Fig. 5d) is consistent with relatively low $\mathrm{CO}$ in the prior when compared to the MOPITT observations. Comparison of the analysis increments, the assimilated MOPITT $\mathrm{CO}$ retrievals, and the independent IASI CO retrievals (lower panels of Figs. 5 and 6) confirms that the assimilation of MOPITT retrievals generally improved the analysis and forecast agreement with the IASI retrievals compared to the MET experiment. Over Baja, MOPITT and to a lesser extent IASI in Fig. 6 report $\mathrm{CO}$ on the order of 50 to $75 \mathrm{ppb}$. The assimilation prior (the $\mathrm{CO}$ forecast) in Fig. 5 has $\mathrm{CO}$ on the order of 125 to $150 \mathrm{ppb}$. The corresponding increment is a $\mathrm{CO}$ reduction on the order of $50 \mathrm{ppb}$. The IASI CO map in Fig. 5 also confirms adjustments over Oklahoma, Kansas, and Nebraska, and to a lesser extent to the east of Georgia, South Carolina, and Virginia.

Figure 7 shows horizontal domain average vertical profiles for the MET and CPSR experiments compared against horizontal domain average profiles for MOPITT and IASI. The WRF-Chem profiles are plotted in retrieval space (after accounting for the averaging kernel and assimilation prior). Comparison of the model and MOPITT profiles (Fig. 7a) shows that the CPSR experiment generally draws the forecast and analysis profiles closer to MOPITT than does the
MET experiment. The error bars are based on the ensemble uncertainty and suggest that those improvements are significant throughout the troposphere. The same comparisons with the IASI profiles (Fig. 7b) show a different result: (i) in the upper troposphere (pressure $(p)<250 \mathrm{hPa}$ ), the MET experiment draws the forecast and analysis profiles closer to IASI than does the CPSR experiment, and (ii) for $p>250 \mathrm{hPa}$, the CPSR experiment draws the profiles closer to IASI. Here again, the error bars suggest that those changes are significant throughout the troposphere. The results from the comparison with IASI highlight the problem, previously discussed for the MOZAIC comparisons in Fig. 2, with assimilating the potentially biased MOPITT CO retrievals. To address that problem, we propose to discard the biased retrievals and assimilate the unbiased truncated retrieval profiles with the extended CPSR method described in Sect. 4.

In summary, this section shows that assimilation of MOPITT CO retrievals improves analysis fit and forecast skill when compared to MOPITT as well as when compared to the independent (not assimilated) IASI and MOZAIC observations. It shows that (i) the CPSR experiment improves the skill when compared to assimilation of raw retrievals (VMRR and L10VMRR) because the phase space transformation reduces the phase space observation errors, and (ii) the CPSR and QOR experiments yield similar results because they account for the observation error cross-covariance contribution in the same way (the diagonalization transform) and because the linearly dependent portion of the transformed retrievals does not contribute to the analysis increment (explicitly with CPSRs and implicitly through the assimilation algorithm for compressed QORs). It also shows that the CPSR experiment did not improve the skill in the lower troposphere near the surface because (i) MOPITT CO profiles with sufficient DOFS to resolve the lower tropospheric CO signal are relatively rare (for this domain and study period), and (ii) an analysis of the impact of the CPSR compression and diagonalization transforms shows that the upper tropospheric CO signal dominates the MOPITT CO sensitivities. Finally, this section shows that in the upper troposphere assimilation of biased MOPITT observations introduced analysis and forecast error relative to the IASI observations.

\subsection{Assimilation of truncated retrieval profiles}

In this section, we test two methods for assimilating truncated retrieval profiles: (i) assimilating L10VMRR retrievals after discarding the biased retrievals (the L10VMRR-RJ3 experiment where the RJ3 indicates that we do not assimilate retrievals above $300 \mathrm{hPa}$ - the upper three levels of the MOPITT CO retrieval profile) and (ii) assimilating CPSRs with the extension to truncated retrieval profiles as described in Sect. 4.3 (the CPSR-RJ3 experiment). The L10VMRR-RJ3 experiment is included only for comparison purposes. If the L10VMRR-RJ3 and CPSR-RJ3 experiments give similar re- 

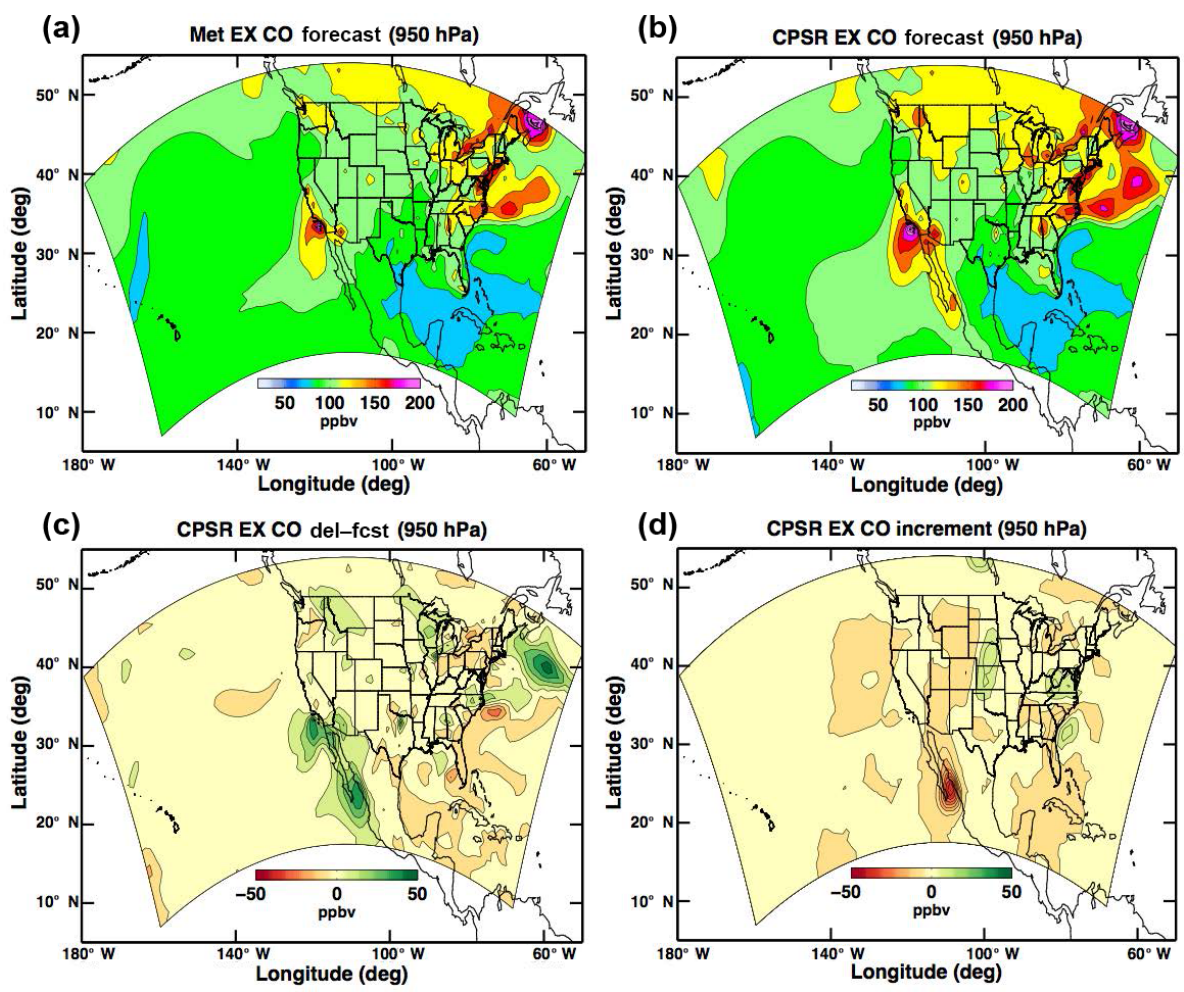

Figure 5. Shaded contours of CO in ppb for the MET and CPSR experiment $6 \mathrm{~h}$ forecasts valid at this cycle time in panels (a) and (b), respectively. Panels (c) and (d) represent the difference between the CPSR and MET forecasts (the CPSR experiment $6 \mathrm{~h}$ forecast minus the MET experiment $6 \mathrm{~h}$ forecast) in panels (a) and (c) and the assimilation increment for analysis at this cycle time in panels (b) and (d). All figures are for $\sim 950 \mathrm{hPa}$ and the 9 June 2008, 18:00 UTC, cycle. The curved rectangle represents the WRF-Chem domain.

sults, then the CPSR-RJ3 approach is preferred because it is computationally less expensive, removes linear dependencies, and accounts for the observation error covariance.

Figure 8 shows vertical profiles for the L10VMRR-RJ3 and CPSR-RJ3 experiments with results from the full retrieval profile assimilation experiments included for reference. In these experiments, we are assuming that the MOPITT retrievals are positively biased in the upper troposphere and the IASI CO retrievals more accurately reflect the true atmospheric state. Comparisons against the assimilated MOPITT observations in the upper panels show that discarding the biased observations had the desired effect - in the upper troposphere, the L10VMRR-RJ3 experiment removes the bias and the analysis profile is drawn closer to that of the MET experiment than in the L10VMRR experiment. Similar results are seen for the CPSR-RJ3 experiment in the last two columns of the upper row. Unexpectedly, for both experiments, not assimilating observations in the upper troposphere had a negative impact in the lower troposphere. A comparison with IASI CO retrievals in the lower row of Fig. 8 shows similar results: (i) the L10VMRR-RJ3 and CPSR-RJ3 retrieval space profiles are drawn closer to the IASI profile than the L10VMRR and CPSR profiles in the upper troposphere, and (ii) the skill is degraded in the middle and lower tropo- sphere. We investigate the cause of those lower tropospheric results later in this section, but first we review the horizontal impacts of the truncated retrieval assimilation experiments.

Figures 9 and 10 show contour maps for the CPSRRJ3 experiment. Figure 9 shows the near-surface impacts, and Fig. 10 shows the upper tropospheric impacts. The $\mathrm{CO}$ $6 \mathrm{~h}$ forecast contour maps in the upper row of Fig. 9 confirm that not assimilating the biased retrievals negatively impacted the lower troposphere because the assimilation impacts are small. The forecast difference maps in the lower row show the impacts in the lower troposphere from assimilating MOPITT CO in the upper troposphere. The CPSR-RJ3 experiment does not have those impacts. It has small large-scale CO decreases over the oceans and eastern United States similar to but weaker than in the CPSR experiment. Also, the magnitude of positive forecast differences at $\mathrm{CO}$ hot spots over southern California, Baja, and the northeastern United States has decreased. Figure 10 shows fewer large-scale changes for the CPSR-RJ3 experiment except for the reductions over the southeastern United States and Gulf of Mexico. Here, the CPSR-RJ3 experiment has large reductions in the $\mathrm{CO}$ adjustments (reducing the bias). Figure 10 provides additional demonstration that discarding the biased retrievals reduces the model's upper tropospheric bias. Unfortunately, we ob- 

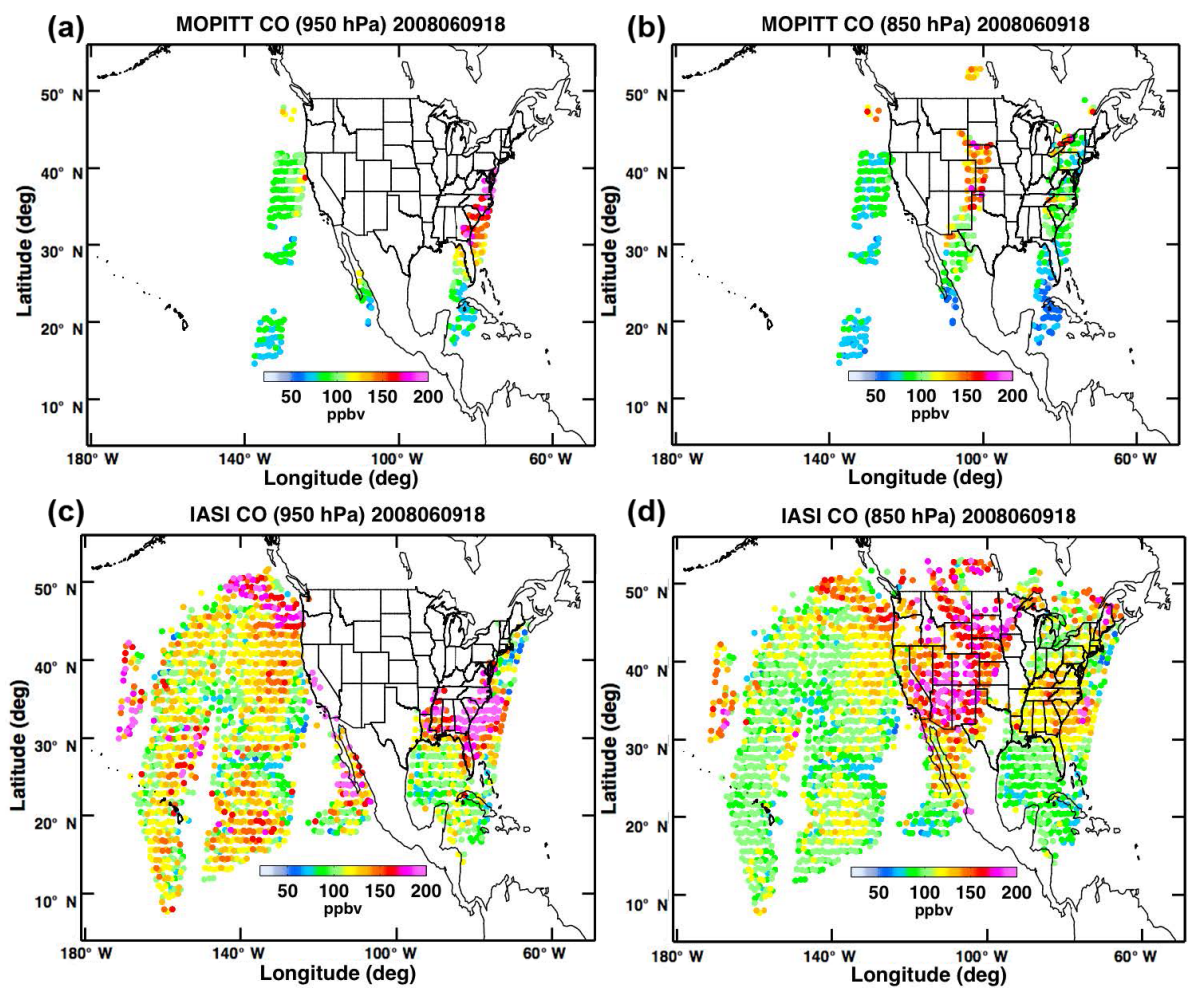

Figure 6. The assimilated MOPITT CO retrievals in panels (a) and (b) and the corresponding IASI CO retrievals (not assimilated) in panels (c) and (d). Panels (a) and (c) are for $\sim 950 \mathrm{hPa}$, and panels (b) and (d) are for $\sim 850 \mathrm{hPa}$. All figures are for the $9 \mathrm{June} 2008$, 18:00 UTC, cycle. The retrievals are in $\mathrm{ppb}$.
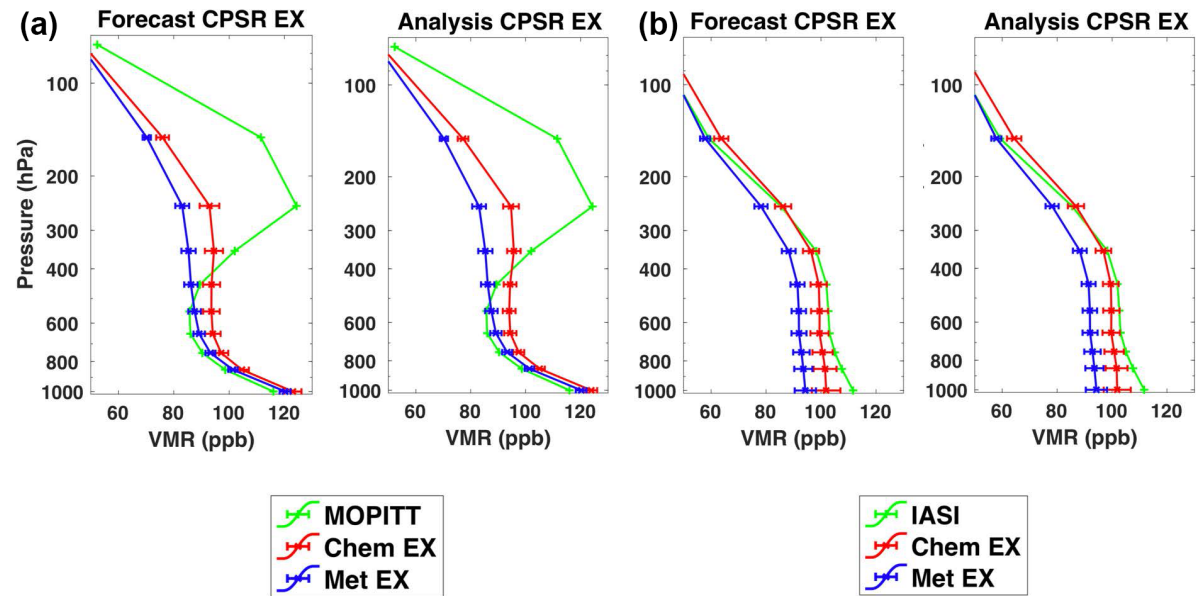

Figure 7. Vertical profiles of the time/horizontal domain average CO in ppb from the CPSR and MET experiments for 9 June 2008 , 18:00 UTC, in retrieval space. "Forecast" is the assimilation prior, and "analysis" is the assimilation posterior. Panel (a) compares the forecast/assimilation results against MOPITT CO retrievals (assimilated), and panel (b) compares those results against IASI CO retrievals (not assimilated). In the legends, Chem EX refers to the CPSR experiment. The error bars are based on the ensemble variability.

tain that result at the expense of reduced improvements in the lower troposphere.

A verification analysis for the L10VMRR-RJ3 and CPSRRJ3 experiments is presented in Fig. 1. The L10VMRRRJ3 and CPSR-RJ3 experiments have degraded forecast skill compared to the full profile assimilation experiments (the CPSR and QOR experiments), but the CPSR-RJ3 experiment has slightly improved skill compared to the L10VMRR-RJ3 experiment. That small improvement is likely due to obser- 

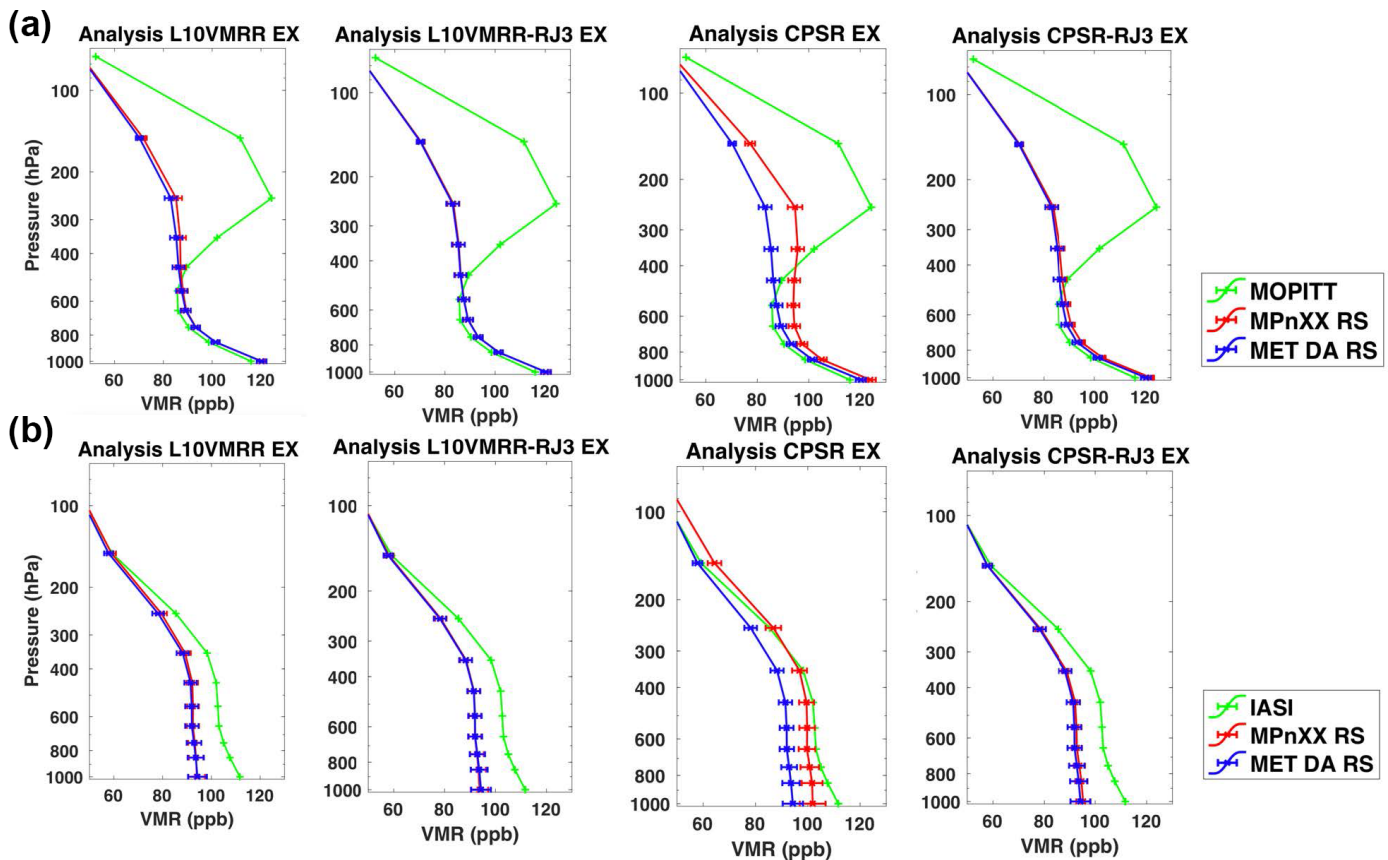

Figure 8. Same as Fig. 7 except this figure compares the L10VMRR, L10VMRR-RJ3, CPSR, and CPSR-RJ3 experiments. Panel (a) compares the forecast/assimilation results against MOPITT CO retrievals (assimilated), and panel (b) compares those results against IASI CO retrievals (not assimilated). In the legends, Chem EX is a placeholder for the L10VMRR-RJ3, L10VMRR, CPSR, and CPSR-RJ3 experiments depending on the panel.

Table 3. Average total and fractional information content for each mode of the averaging kernel for the entire study period. CompAK 1 denotes the average fractional information in mode 1, CompAK 2 is for mode 2, and so forth. Trace denotes the total information content. "Full profile" means all retrievals were assimilated (i.e., none were discarded). "Reject top three" means that retrievals at pressure levels $<300 \mathrm{hPa}$ were discarded. "Reject middle three" means that retrievals between 300 and $600 \mathrm{hPa}$ were discarded. "Reject bottom three" means that retrievals below $700 \mathrm{hPa}$ were discarded.

\begin{tabular}{lrrrr}
\hline & CompAK 1 & CompAK 2 & CompAK 3 & Trace \\
\hline Full profile & 0.9638 & 0.4785 & 0.0099 & 1.452 \\
Reject top three & 0.7983 & 0.2851 & 0.0045 & 1.088 \\
Reject middle three & 0.7254 & 0.3849 & 0.0078 & 1.118 \\
Reject bottom three & 0.9335 & 0.3770 & 0.0065 & 1.317 \\
\hline
\end{tabular}

vation error covariance reductions from the CPSR transform as discussed earlier.

In summary, not assimilating the biased observations had positive impacts in the upper troposphere and negative impacts in the middle to lower troposphere. We suspect the negative results occurred for two reasons. Discarding retrievals and their averaging kernels (i) reduces the total information content of the assimilated retrievals so that the assimilation adjustments are small; and (ii) reduces the sensitivity of the transformed averaging kernel so that the expected retrievals are less sensitive to the true atmospheric profile. Those reductions combine to reduce the ensemble state variable cor- relations and consequently the assimilation impacts. To test explanation (i), we compare the trace of the composited raw averaging kernel for the CPSR experiment with that for the CPSR-RJ3 experiment. The results are shown in the first two rows of Table 3 where "full profile" is from the CPSR experiment, and "reject top three" is from the CPSR-RJ3 experiment. Comparison of those results shows a $25 \%$ reduction in the trace indicating that the total information content of the assimilated retrievals for the CPSR-RJ3 experiment is $25 \%$ less than that for the CPSR experiment. For comparison purposes, Table 3 also shows trace reductions from not assimilating retrievals in the middle troposphere ( $23 \%$ reduction) and lower troposphere ( $9 \%$ reduction). Those results suggest that most of the information in the MOPITT CO retrievals is from the upper troposphere, the second greatest amount is from the middle troposphere, and the smallest amount is from the lower troposphere. To test explanation (ii), we plot the compressed and fully transformed averaging kernels in Fig. 11 where column (a) is for the CPSR experiment and column (b) is for the CPSR-RJ3 experiment. Figure 11 is similar to the last two rows of Fig. 4. Recall that the first row represents the sensitivity of the compressed QORs to the true $\mathrm{CO}$ concentrations, and the second row represents the sensitivity of the CPSRs to the true CO concentrations. Comparison of columns (a) and (b) shows that for the CPSR-RJ3 experiment, the leading mode sensitivities are reduced when compared to the CPSR experiment. The state variable corre- 

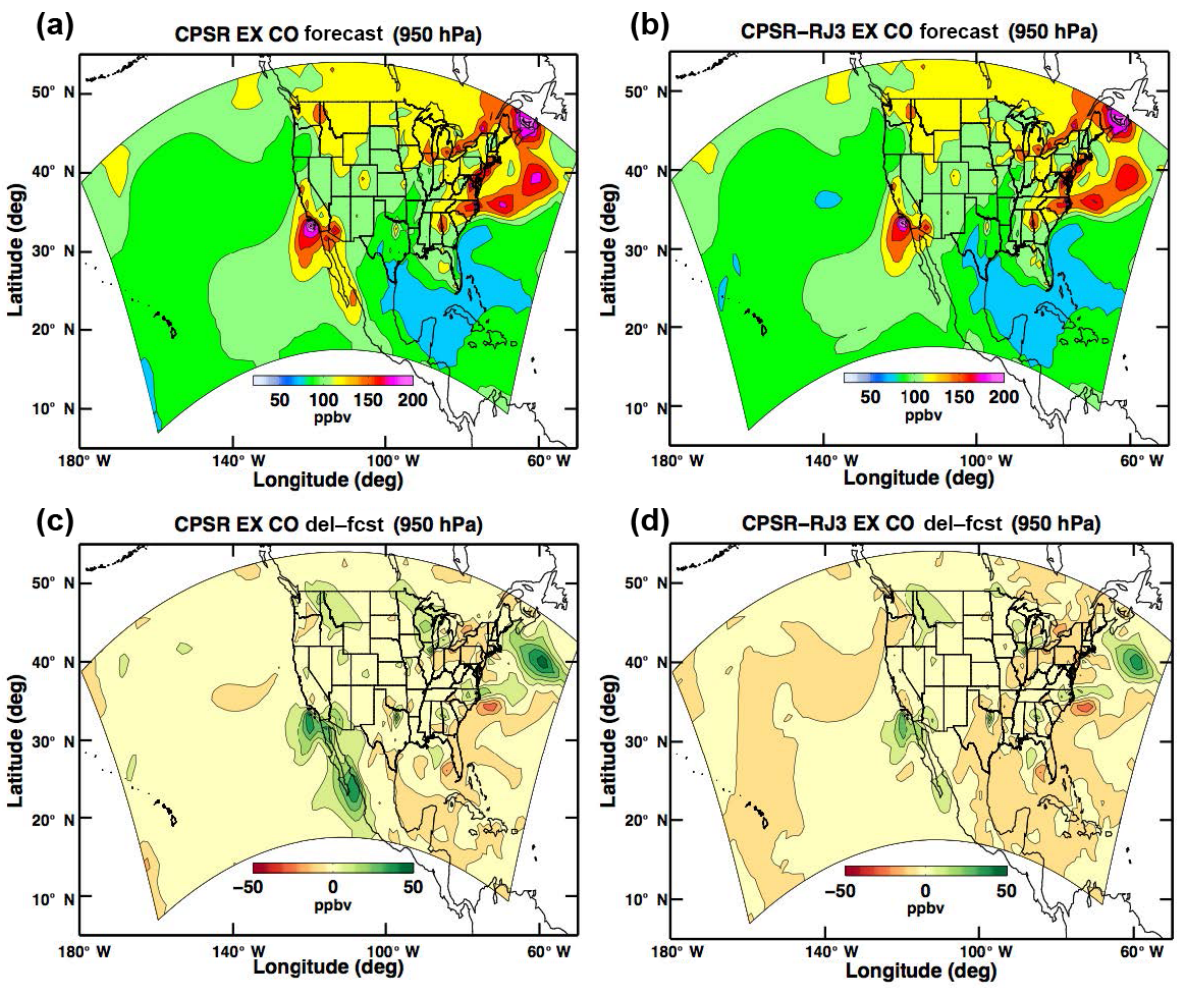

Figure 9. Shaded contours of CO in ppb for the CPSR and CPSR-RJ3 experiment assimilation priors in panels (a) and (b), respectively, and for the CPSR and MET experiment difference (the CPSR minus the MET experiment, defined as CPSR EX CO Del-Fcst) and the CPSR-RJ3 and MET experiment difference (the CPSR-RJ3 minus the MET experiment, defined as CPSR-RJ3 EX CO Del-Fcst) assimilation priors in panels (c) and (d), respectively. The CPSR experiments maps in this figure are the same as in Fig. 5 and included for reference. All figures are for $\sim 950 \mathrm{hPa}$ on 9 June 2008, 18:00 UTC.

lations are proportional to those sensitivities, so the reduced correlations result in analysis increment reductions. For comparison purposes, columns (c) and (d) of Fig. 11 show results from experiments that discard retrievals in the middle and lower troposphere. Those profiles, in combination with Table 2, show that most of the information and sensitivity is associated with the upper and midtropospheric retrievals. Discarding upper tropospheric retrievals alters the sensitivity magnitudes while discarding middle tropospheric retrievals alters the magnitudes and vertical structure. One interesting result is that most of the sensitivity loss in column (c) - the "reject middle three" experiment - appears to be associated with the CPSR diagonalization transform. That suggests that the sensitivity loss is dependent on specification of the retrieval a priori error covariance.

Those changes occur because as different rows of the averaging kernel are discarded (i) the amount of observed information in the modified averaging kernel changes, and (ii) the vertical structure of the bases for the range and domain of the modified averaging kernel changes. The impact of changes in the information content in point (i) were discussed earlier. The impact of changes to the bases in point (ii) has important consequences. The leading left singular vectors of the trans- formed averaging kernel span the range of the transformed averaging kernel but their vertical structure and possibly their dimension change when retrievals are discarded. That means the phase space observations change because the basis vectors used in the compression transform are different, and their sensitivity to the truncated retrieval profile vector is different. Similarly, the leading right singular vectors of the transformed averaging kernel span the domain of the transformed averaging kernel, but their vertical structure changes when retrievals are discarded. Those changes occur solely because the information content of the transformed averaging kernel is reduced (since the dimension of its domain - the space where the true CO profiles reside - is unchanged). Those changes are significant because they alter the elements (or levels) of the true profile to which the transformed averaging kernel is sensitive. To summarize, not assimilating elements of the full retrieval profile alters the levels of the retrieval profile to which the phase space observations are sensitive. Discarding those elements also alters the levels of the true $\mathrm{CO}$ profile to which the transformed averaging kernel is sensitive. Those sensitivity changes occur regardless of whether the assimilation is done in phase space as in the CPSR-RJ3 experiment or in retrieval space as in L10VMRR-RJ3 exper- 

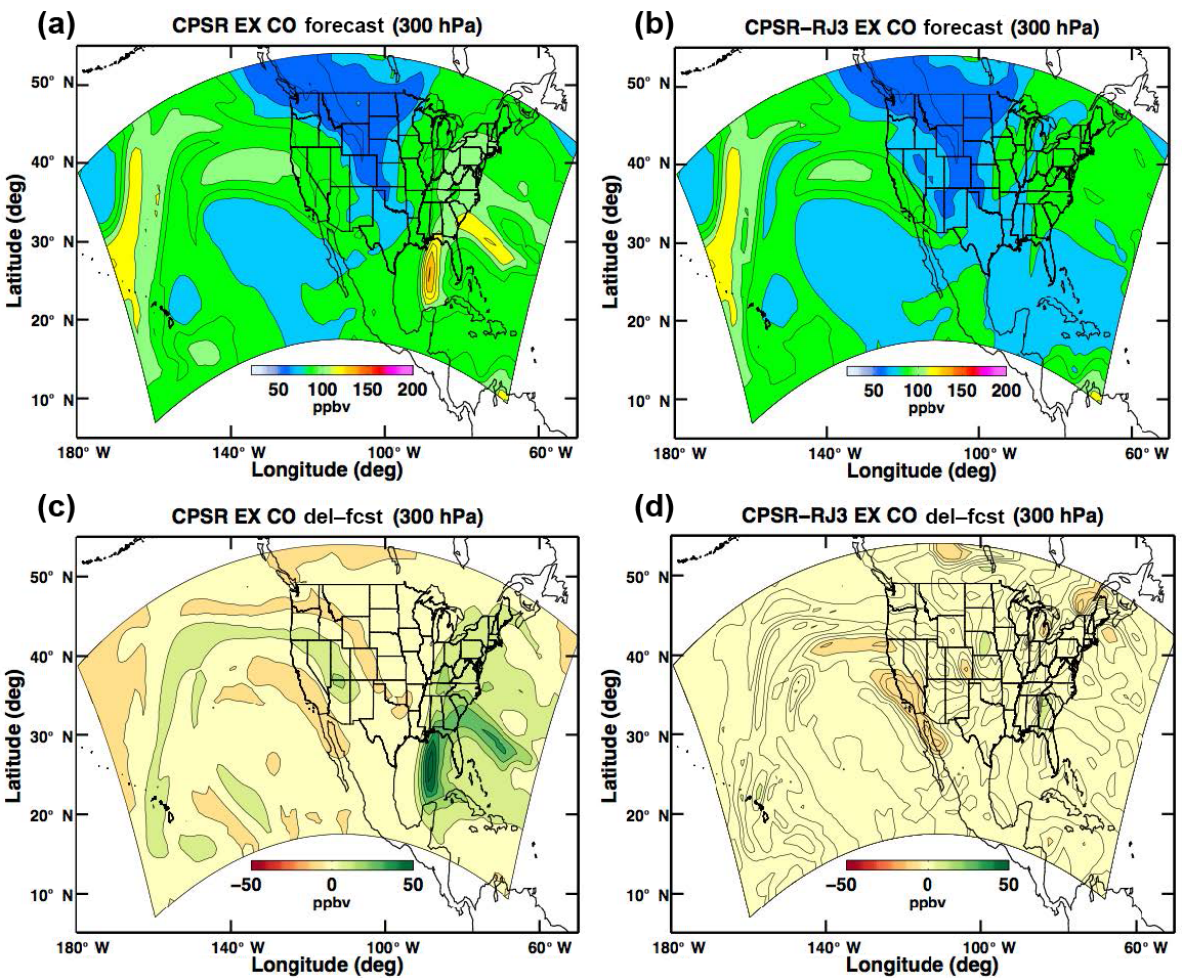

Figure 10. Same as Fig. 9 except for $\sim 300 \mathrm{hPa}$.

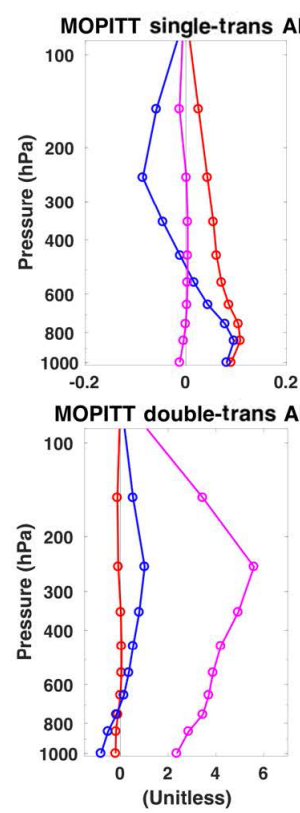

(a)
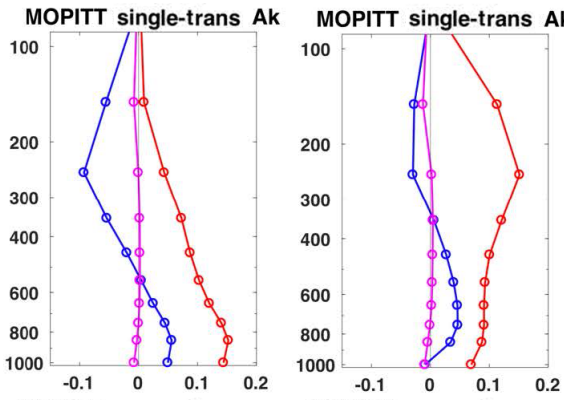

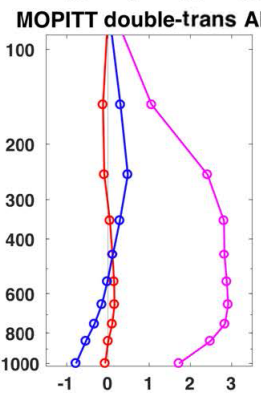

(b)

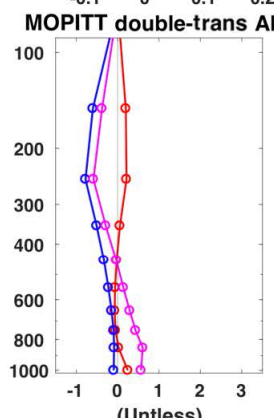

(c)
MOPITT single-trans Ak
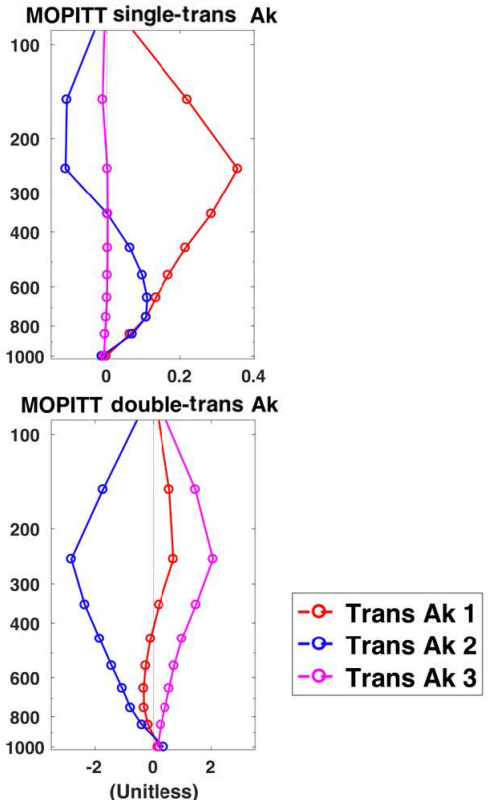

(d)

Figure 11. Same as the lower two rows of Fig. 4 except that this figure is for the retrieval discard experiments. Column (a) is for the full retrieval profile assimilation experiment and is the same as column (a) in Fig. 3. Column (b) is for the "reject top three" experiment in Table 2. Column (c) is for the "reject middle three" experiment. Column (d) is for the "reject bottom three" experiment. Notice that the range of the abscissa is reduced from column (a) to columns (b-d). 
iment. Consequently, results from the L10VMRR-RJ3 and CPSR-RJ3 experiments are similar.

This section shows that CPSRs can be extended to the assimilation of truncated retrieval profiles but that discarding upper tropospheric observations for MOPITT significantly reduces the total information content of the assimilated observations and the vertical sensitivity of the transformed averaging kernel profiles. Those reductions translate to reductions in the state variable correlations and commensurate reductions in the analysis increments. We are studying modification of the CPSR extension to truncated retrieval profiles to address the non-local impacts.

\section{Summary and conclusions}

This paper had two goals: (i) compare the results from assimilating CPSRs with independent observations (we used MOZAIC in situ observations and IASI CO retrievals as the independent observations), and (ii) extend CPSRs to the assimilation of truncated retrieval profiles. The comparison with independent observations showed that (i) assimilation of raw retrievals (VMRRs and L10VMRRs) had little impact on the analysis fit and forecast skill due to the magnitude of the observation errors and the length of the study period, and (ii) the assimilation of phase space retrievals (CPSRs and QORs) improved both fit and skill. Conceptually, we expect the assimilation of raw retrievals and phase space retrievals to yield similar results. However, phase space transformation of the observation error covariance truncated the observation errors so that the CPSR and QOR experiments produced closer agreement with the assimilated and independent observations. This does not mean that the assimilation of raw retrievals is incorrect. It means only that the reported observations errors may be too large because they account for errors associated with the retrieval prior and consequently they require a longer study period to show an assimilation impact compared to CPSRs.

Comparison of the CPSR experiments with IASI CO retrievals and MOZAIC in situ CO observations generally showed improved agreement in the middle and lower troposphere compared to the MET experiment. For the IASI comparison, the improvements were significant and extended from $250 \mathrm{hPa}$ to the surface. For the MOZAIC comparison, two (Dallas, TX and Portland, OR) of the three (no improvement for Philadelphia, PA) urban areas studied showed improvements between 500 and $800 \mathrm{hPa}$. Below $800 \mathrm{hPa}$, there was little to no improvement. Although the assimilation impacts when compared to MOZAIC were not significant, the lack of a near-surface improvement was unexpected. However, the DOFS analysis in the discussion of Figs. 3 and 4 showed that there were no near-surface impacts because after accounting for the observation error covariance, the transformed averaging kernel had very little sensitivity to the nearsurface $\mathrm{CO}$. Other researchers have not found that result be- cause they have not accounted for the observation error correlations.

Comparison of the CPSR experiment with IASI and MOZAIC showed degraded skill in the upper troposphere (above $250 \mathrm{hPa}$ for IASI and above $500 \mathrm{hPa}$ for MOZAIC) compared to the MET experiment. That degradation was significant for IASI but not MOZAIC. It was attributed to the assimilation of biased retrievals above $300 \mathrm{hPa}$ illustrating the need to extend the CPSR method to truncated retrieval profiles. Section 4.3 explained the extension, and Sect. 5.2 compared the L10VMRR-RJ3 (assimilation of truncated raw retrieval profiles) and CPSR-RJ3 (assimilation of truncated phase space retrieval profiles) experiments where we did not assimilate the biased MOPITT CO retrievals above $300 \mathrm{hPa}$. That comparison showed that the L10VMRR-RJ3 and CPSR-RJ3 experiments produced similar results, confirming the applicability of the CPSR approach to truncated retrieval profiles. However, they also highlighted an important characteristic of assimilating truncated retrieval profiles. Excluding the assimilation of some elements of the observation profiles can significantly alter the (i) information content of the assimilated observations and (ii) the amplitude of the averaging kernel sensitivities. Those modifications can combine to reduce the state variable correlations and the corresponding analysis increments. We are researching modification of the CPSR extension to truncated retrieval profiles to address the reduced impact from not assimilating retrievals from selected levels.

Code and data availability. The current versions of the WRFChem, WRF, WRFVAR, and WPS codes are available from the WRF download site at http://www2.mmm.ucar.edu/wrf/users/ download/get_sources.html (last access: 6 September 2018). The current version of the DART code is at available at https://www.image.ucar.edu/DAReS/DART/DART2_Starting. php\#download (last access: 6 September 2018), and the current version of the WRF-Chem/DART branch is available at http://www.image.ucar.edu/DAReS/DART/DART_download (last access: 6 September 2018). The WRF-Chem/DART branch is the same as the DART code except for the inclusion of the WRFChem/DART system. There is no need to download both codes. Presently, there is no user guide available for WRF-Chem/DART. However, the authors have prepared a slide presentation that describes much of the chemical data assimilation script function, variables, and organization. Interested readers should contact the first author for a copy of that presentation and assistance with using WRF-Chem/DART. The large-scale model's forecast and observational data used to run the ensemble forecast/data assimilation cycling experiments described in the paper are generally available from the respective data distribution sites. That data set has not been posted to a public site due to its size but is available from the first author upon request.

Author contributions. As the primary author, APM was responsible for conducting the research and writing the paper. His subject matter 
contributions were developing and maintaining WRF-Chem/DART, developing and advancing "compact phase space retrievals", and ensemble chemical data assimilation. As secondary authors, DPE and JLA were research mentors, collaborators, and reviewers and provided guidance on research direction. DPE's expertise is generally satellite retrieval systems and specifically the MOPITT CO retrieval system. JLA's expertise is generally ensemble assimilation of meteorological observations. He, along with his research group at NCAR/DAReS, is responsible for developing and maintaining DART.

Competing interests. The authors declare that they have no conflict of interest.

Acknowledgements. NCAR is sponsored by the National Science Foundation (NSF). Any opinions, findings, and conclusions or recommendations expressed in this publication are those of the authors and do not necessarily reflect the view of NSF. This research is also sponsored by National Aeronautics and Space Administration (NASA) grant NNX11AI51G. We gratefully acknowledge Chris Snyder and Avellino Arellano for discussions that led to a better understanding of the transformed averaging kernels associated with truncated retrieval profiles. We also acknowledge Louisa Emmons and Benjamin Gaubert for their thoughtful reviews and helpful suggestions, both of which improved the quality of the manuscript. We also acknowledge the use of data products from the MOPITT, IASI, and MOZAIC/IAGOS programs.

Edited by: Jason Williams

Reviewed by: two anonymous referees

\section{References}

Anderson, J. L.: An ensemble adjustment Kalman filter for data assimilation, Mon. Weather Rev., 129, 2884-2903, https://doi.org/10.1175/15200493(2001129<2884:AEAKFF>2.CO:2, 2001.

Anderson, J. L.: A local least squares framework for ensemble filtering, Mon. Weather Rev., 131, 634-642, https://doi.org/10.1175/15200493(2003)<0634:ALLSFF>2.0.CO:2, 2003.

Anderson, J. L.: Spatially and temporally varying adaptive covariance inflation for ensemble filters, Tellus, 61, 72-83, https://doi.org/10.1111/j.1600-0870.2008.00361.x, 2008.

Anderson, J. L., Hoar, T., Raeder, K., Liu, H., Collins, N., Torn, R., and Arellano, A.: The Data Assimilation Research Testbed: A community facility, B. Am. Meteorol. Soc., 90, 1283-1296, https://doi.org/10.1175/2009BAMS2618.1, 2009.

Arellano Jr., A. F., Raeder, K., Anderson, J. L., Hess, P. G., Emmons, L. K., Edwards, D. P., Pfister, G. G., Campos, T. L., and Sachse, G. W.: Evaluating model performance of an ensemble-based chemical data assimilation system during INTEX-B field mission, Atmos. Chem. Phys., 7, 5695-5710, https://doi.org/10.5194/acp-7-5695-2007, 2007.

Barker, D., Huang, X.-Y., Liu, Z., Auligné, T., Zhang, X., Rugg, S., Ajjaji, R., Bourgeois, A., Bray, J., Chen, Y., Demirtas, M.,
Guo, Y.-R., Henderson, T., Huang, W., Lin, H.-C., Michalakes, J., Rizvi, S., and Zhang, X.: The Weather Research and Forecasting Model's Community Variational/Ensemble Data Assimilation System: WRFDA, B. Am. Meteorol. Soc., 93, 831-843, https://doi.org/10.1175/BAMS=D-11-00167.1, 2012.

Barre, J., Gaubert, B., Arellano, A. F., Worden, H. M., Edwards, D. P., Deeter, M. N., Anderson, J. L., Raeder, K., Collins, N., Tilmes, S., Francis, G., Clerbaux, C., Emmons, L. K., Pfister, G. G., Coheur, P.-F., and Hurtmans, D.: Assessing the impacts of assimilating IASI and MOPITT CO retrievals using CESMCAM-chem and DART, J. Geophys. Res.-Atmos., 120, 1050110529, https://doi.org/10.1002/2015JD023467, 2015.

Clerbaux, C., Boynard, A., Clarisse, L., George, M., Hadji-Lazaro, J., Herbin, H., Hurtmans, D., Pommier, M., Razavi, A., Turquety, S., Wespes, C., and Coheur, P.-F.: Monitoring of atmospheric composition using the thermal infrared IASI/MetOp sounder, Atmos. Chem. Phys., 9, 6041-6054, https://doi.org/10.5194/acp-96041-2009, 2009.

Deeter, M. N., Edwards, D. P., and Gille, J. C.: Retrievals of carbon monoxide profiles from MOPITT observations using lognormal a priori statistics, J. Geophys. Res., 112, D11311, https://doi.org/10.1029/2006JD007999, 2007a.

Deeter, M. N., Edwards, D. P., Gille, J. C., and Drummond, J. R.: Sensitivity of MOPITT observations to carbon monoxide in the lower troposphere, J. Geophys. Res., 112, D24306, https://doi.org/10.1029/2007JD008929, 2007 b.

Deeter, M. N., Martinez-Alonso, S., Edwards, D. P., Emmons, L. K., Gille, J. C., Worden, H. M., Pittman, J. V., Daube, B. C., and Wofsy, S. C.: Validation of MOPITT Version 5 thermalinfrared, near-infrared, and multispectral carbon monoxide profile retrievals for 2000-2011, J. Geophys. Res.-Atmos., 118, 6710-6725, https://doi.org/10.1002/jgrd.50272, 2013.

Deeter, M. N., Martínez-Alonso, S., Edwards, D. P., Emmons, L. K., Gille, J. C., Worden, H. M., Sweeney, C., Pittman, J. V., Daube, B. C., and Wofsy, S. C.: The MOPITT Version 6 product: algorithm enhancements and validation, Atmos. Meas. Tech., 7, 3623-3632, https://doi.org/10.5194/amt-7-3623-2014, 2014.

Eskes, H. J. and Boersma, K. F.: Averaging kernels for DOAS totalcolumn satellite retrievals, Atmos. Chem. Phys., 3, 1285-1291, https://doi.org/10.5194/acp-3-1285-2003, 2003.

Harvey, C.: The Staggering economic cost of air pollution, Washington Post, Energy and Environment, https://www.washingtonpost.com/news/energy-environment/ wp/2016/01/29/the-staggering-economic-cost-of-air-pollution/ ?noredirect $=$ on\&utm_term $=. c 25 \mathrm{a} 8 \mathrm{cfa} 222 \mathrm{~b}$ (last access: 6 September 2018), 29 January 2016.

Jiang, Z., Jones, D. B. A., Worden, J., Worden, H. M., Henze, D. K., and Wang, Y. X.: Regional data assimilation of multispectral MOPITT observations of CO over North America, Atmos. Chem. Phys., 15, 6801—6814, https://doi.org/10.5194/acp15-6801-2015, 2015.

Joiner, J. and Da Silva, A. M.: Efficient methods to assimilate remotely sensed data based on information content, Q. J. Roy. Meteor. Soc., 124, 1669-1694, https://doi.org/10.1002/qj.49712454915, 2006.

Marenco, A., Thouret, V., Nedelec, P., Smit, H., Helten, M., Kley, D., Karcher, F., Simon, P., Law, K., Pyle, J., Poschmann, G., Von Wrede, R., Hume, C., and Cook, T., Measurement of ozone and water vapor by Airbus in-service aircraft: The MOZAIC 
airborne program, an overview, J. Geophys. Res., 103, 2563125642, https://doi.org/10.1029/98jD00977, 1998.

Martinez-Alonso, S., Deeter, M. N., Worden, H. M., Gille, J. C., Emmons, L. K., Pan, L. L., Park, M., Manney, G. L., Bernath, P. F., Boone, C. D., Walker, K. A., Kolonjari, F., Wofsy, S. C., Pittman, J., and Daube, B. C., Comparison of upper tropospheric carbon monoxide from MOPITT, ACD-FTC, and HIPPO-QCLS, J. Geophys. Res.-Atmos., 119, 14164-14164, https://doi.org/10.1002/2014JD022397, 2014.

Migliorini, S., Piccolo, C., and Rodgers, C. D.: Use of the information content in satellite measurements for an efficient interface to data assimilation, Mon. Weather Rev., 136, 2633-2650, 2008.

Miyazaki, K., Eskes, H. J., and Sudo, K.: Global $\mathrm{NO}_{x}$ emission estimates derived from an assimilation of OMI tropospheric $\mathrm{NO}_{2}$ columns, Atmos. Chem. Phys., 12, 2263-2288, https://doi.org/10.5194/acp-12-2263-2012, 2012a.

Miyazaki, K., Eskes, H. J., Sudo, K., Takigawa, M., van Weele, M., and Boersma, K. F.: Simultaneous assimilation of satellite $\mathrm{NO}_{2}$, $\mathrm{O}_{3}, \mathrm{CO}$, and $\mathrm{HNO}_{3}$ data for the analysis of tropospheric chemical composition and emissions, Atmos. Chem. Phys., 12, 95459579, https://doi.org/10.5194/acp-12-9545-2012, 2012b.
Miyazaki, K., Eskes, H. J., and Sudo, K.: A tropospheric chemistry reanalysis for the years 2005-2012 based on an assimilation of OMI, MLS, TES, and MOPITT satellite data, Atmos. Chem. Phys., 15, 8315-8348, https://doi.org/10.5194/acp15-8315-2015, 2015.

Mizzi, A. P., Arellano Jr., A. F., Edwards, D. P., Anderson, J. L., and Pfister, G. G.: Assimilating compact phase space retrievals of atmospheric composition with WRFChem/DART: a regional chemical transport/ensemble Kalman filter data assimilation system, Geosci. Model Dev., 9, 965-978, https://doi.org/10.5194/gmd-9-965-2016, 2016.

Robichaud, A.: Surface data assimilation of chemical compounds over North America and its impact on air quality and Air Quality Health Index (AQHI) forecasts, Air Qual. Atmos. Health, 10, 955-970, https://doi.org/10.2017/s11869-017-0485-9, 2017. 